\title{
An Extension of the Birnbaum-Saunders Distribution Based on Skew-Normal-t Distribution
}

\author{
Farzane Hashemi, Vahid Amirzadeh and Ahad Jamalizadeh* \\ Shahid Bahonar University of Kerman
}

\begin{abstract}
In this paper, we introducte a family of univariate BirnbaumSaunders distributions arising from the skew-normal- $t$ distribution. We obtain several properties of this distribution such as its moments, the maximum likelihood estimation procedure via an EM-algorithm and a method to evaluate standard errors using the EM-algorithm. Finally, we apply these methods to a real data set to demonstrate its flexibility and conduct a simulation study to demonstrate the usefulness of this distribution when compared to the ordinary Birnbaum-Saunders and skew-normal Birnbaum-Saunders distributions.
\end{abstract}

Keywords. EM and ECM algorithms; Monte Carlo simulations; observed information matrix; stochastic representation.

MSC 2010: 62E10, 62F10, 62G30.

\section{Introduction}

The Birnbaum-Saunders $(B S)$ distribution was originally introduced by Birnbaum and Saunders (1969) as a model for the distribution of a unit under cyclic loading. This distribution is obtained on the basis of the standard normal distribution by means of a random variable (r.v.) $T$ which admits

* Corresponding author 
the stochastic representation

$$
T \stackrel{d}{=} \frac{\beta}{4}\left[\alpha Z+\sqrt{(\alpha Z)^{2}+4}\right]^{2},
$$

where $Z \sim N(0,1)$. The probability density function (pdf) of $T$ is given by

$$
g(t ; \alpha, \beta)=\phi(a(t ; \alpha, \beta)) A(t ; \alpha, \beta), \quad t, \alpha, \beta>0,
$$

where

$$
a(t ; \alpha, \beta)=\frac{1}{\alpha}\left[\sqrt{\frac{t}{\beta}}-\sqrt{\frac{\beta}{t}}\right], \quad A(t ; \alpha, \beta)=\frac{d a(t ; \alpha, \beta)}{d t}=\frac{t+\beta}{2 \alpha \sqrt{\beta} \sqrt{t^{3}}}
$$

and $\phi(\cdot)$ is the pdf of the standard normal distribution. We say that $T$ follows the BS distribution with shape parameter $\alpha>0$ and scale parameter $\beta>0$.

The $B S$ distribution is a popular model, commonly used in different areas of sciences and engineering; for example, Desmond (1985) considered the $B S$ distribution as a model in biology, Leiva et al. (2007), Barros et al. (2008) presented some applications in the medical field, and Podlaski (2008), Leiva et al. (2010) and Vilca et al. (2010) used it to model data related to the forestry and environmental sciences.

Recently researchers have suggested replacing the standard normal variable $Z$ in (1) by other random variables. For instance, Díaz-García and Leiva-Sánchez (2005) introduced the generalized Birnbaum-Saunders $(G B S)$ distribution which replaces the normal distribution by the elliptical family of distributions. The main motivation for the use of the $G B S$ distribution is to make it more flexible. Sanhueza et al. (2008) have presented a detailed review of the results of the $G B S$ distribution and Gómez et al. (2009) have proposed an extension using the slash-elliptical distribution. Other extensions include the $\beta$-Birnbaum-Saunders distribution in Cordeiro and Lemonte (2011), the Marshall-Olkin extended BS distribution in Lemonte (2013) and the non-central version of the $B S$ distribution in Guiraud et al. (2009).

Recently, skewed distributions have received a great deal of attention in the literature and include the family of skew-normal distributions discussed formally and popularized by Azzalini (1985, 1986), as a special case. Let $\Phi(\cdot)$ denote the standard normal $(N(0,1))$ cumulative distribution function (cdf). Then, a random variable $Z$ is said to follow a standard skew-normal (SN) distribution with shape parameter $\lambda \in \mathbb{R}(Z \sim S N(\lambda))$, if its pdf is given by

$$
f_{S N}(z ; \lambda)=2 \phi(z) \Phi(\lambda z), \quad z \in \mathbb{R}
$$


Vilca and Leiva (2006) replaced the r.v. $Z$ in (1) by a $S N$ variable having pdf (2) and obtained the skew-normal Birnbaum-Saunders $(S N-B S)$ distribution with the pdf

$$
\varphi(t ; \alpha, \beta, \lambda)=2 \phi(a(t ; \alpha, \beta)) \Phi(\lambda a(t ; \alpha, \beta)) A(t ; \alpha, \beta), \quad t, \alpha, \beta>0, \lambda \in \mathbb{R} .
$$

Vilca et al. (2011) showed that the $S N-B S$ distribution model is able to predict extreme percentiles. Furthermore, these authors used an EM-type algorithm to obtain the maximum likelihood (ML) estimation of the SN-BS distribution parameters.

Various extensions of the $S N$ distribution, which provide greater flexibility have been suggested in the literature. For example, Nadarajah and Kotz (2003) proposed the skew-normal- $t(S N T)$ distribution . In this paper, replacing the variable $Z$ in (1) by a $S N T$ variable, we introduce a new extension of the $B S$ distribution based on the $S N T$, which we call the skew-normal- $t$ Birnbaum-Saunders $(S N T-B S)$ distribution. We then use a stochastic representation for $S N T$ variable to obtain the ML estimation of the $S N T-B S$ distribution parameters using an EM-type algorithm.

The rest of this paper is organised as follows. In Section 2, a new family of distributions is defined and its properties are studied in details. Section 3 discusses an EM-type algorithm to obtain the parameter estimates of the $S N T-B S$ model. A method to evaluate the standard errors from the EMtype algorithm is also presented. In Section 4 , a real data set is used an illustrate an application of the $S N T-B S$ model. In Section 5, a simulation study is conducted to compare the $B S, S N-B S$ and $S N T-B S$ models, and Monte Carlo simulations are used to evaluate the finite-sample performance of the ML estimates of the $S N T-B S$ distribution parameters.

\section{The Birnbaum-Saunders Distribution Based on Skew-Normal- $t$ Distribution}

We begin this section by defining the $S N T$ distribution and give a stochastic representation of the $B S$ distribution based on a $S N T$ variable. We then derive some properties of the latter distribution as well as its hierarchical formulation.

The random variable $Z$ follows a skew-normal- $t$ distribution with skewness parameter $\lambda \in \mathbb{R}$ and degrees of freedom parameter $\nu \in \mathbb{R}^{+}(S N T(\lambda, \nu))$, 
if its pdf is given by (Nadarajah and Kotz, 2003)

$$
f_{S N T}(z ; \lambda, \nu)=2 \phi(z) T(\lambda z ; \nu), \quad z \in \mathbb{R},
$$

where $T(\cdot ; \nu)$ is the cdf of the Student's $t$ distribution with degrees of freedom $\nu$. Density (4) includes the normal distribution $(\lambda=0)$, and the half normal distribution $(\lambda \rightarrow \infty)$ as special cases. In addition, as $\nu \rightarrow \infty$, (4) becomes the Azzalini's skew normal density.

The following result, due to Azzalini and Capitanio (2003), is useful for evaluating underlying integrals in the rest of the paper:

Propoition 1. If $\tau$ follows gamma distribution with shape parameter $\omega_{1}>0$ and scale parameter $\omega_{2}>0\left(\Gamma\left(\omega_{1}, \omega_{2}\right)\right)$, then for all $a \in \mathbb{R}$,

$$
E(\Phi(a \sqrt{\tau}))=T\left(a \sqrt{\frac{\omega_{1}}{\omega_{2}}} ; 2 \omega_{1}\right) .
$$

In the following proposition we present a stochastic representation for the random variable $Z$ with pdf (4).

Proposition 2. We have

$$
Z \stackrel{d}{=} \frac{1}{\sqrt{1+\lambda^{2} \tau}} Y_{1}+\lambda \sqrt{\frac{\tau}{1+\lambda^{2} \tau}}\left|Y_{0}\right| \sim \operatorname{SNT}(\lambda, \nu),
$$

where $Y_{0}$ and $Y_{1}$ are two independent $N(0,1)$ random variables and $\tau \sim$ $\Gamma(\nu / 2, \nu / 2)$ is independent of $Y_{0}$ and $Y_{1}$.

Proof. See Appendix A.

Definition 1. If $Z \sim S N T(\lambda, \nu)$ in (1), we say that the r.v. $T$ follows the $S N T-B S$ distribution. In this case, the pdf of $T$ is

$$
\begin{aligned}
f_{T}(t ; \alpha, \beta, \lambda, \nu)= & 2 \phi(a(t ; \alpha, \beta)) T(\lambda a(t ; \alpha, \beta) ; \nu) \\
& \times A(t ; \alpha, \beta), \quad t, \alpha, \beta, \nu>0, \lambda \in \mathbb{R} .
\end{aligned}
$$

Figure 1 displays the graphs of $B S, S N-B S$ and $S N T-B S$ densities, for selected values of parameters. Note that the figure shows better fit of lower percentiles for the $S N T-B S$ distribution related to the $B S$ and 

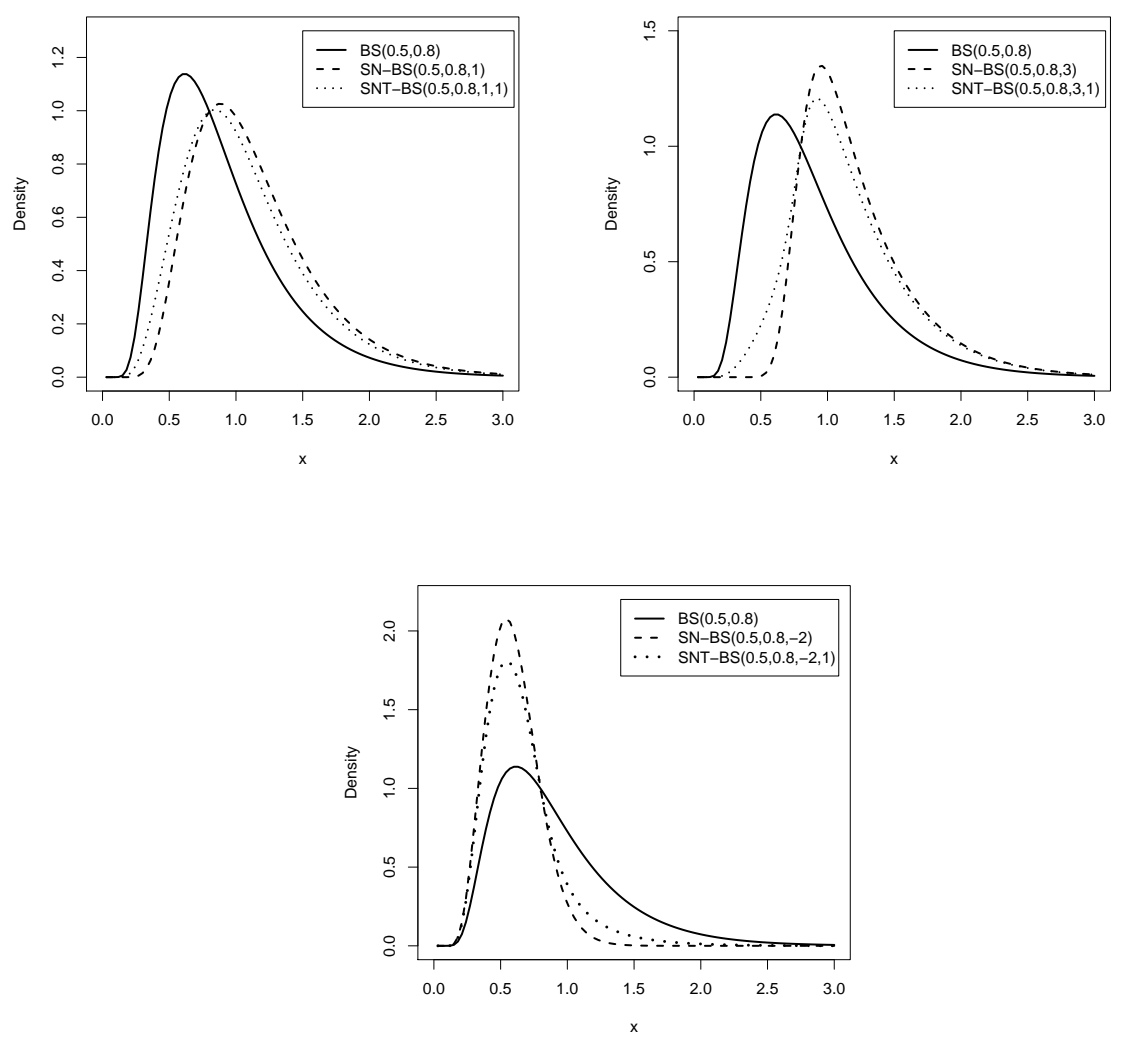

Figure 1. Plots of the $B S, S N-B S$ and $S N T-B S$ densities for selected values of parameters.

$S N-B S$ distributions.

Some properties of the $S N T-B S(\alpha, \beta, \lambda, \nu)$ distribution are as follows:

(i) For $\lambda=0$, the $S N T-B S$ distribution coincides with the $B S(\alpha, \beta)$ distribution.

(ii) When $\nu \rightarrow \infty$, the pdf (7) approaches the pdf in equation (3).

(iii) The pdf (7) tends to $2 g(t ; \alpha, \beta)$, as $\lambda \rightarrow \infty$.

(iv) If $T \sim S N T-B S(\alpha, \beta, \lambda, \nu)$ then $T^{-1} \sim S N T-B S\left(\alpha, \beta^{-1}, \lambda, \nu\right)$. 
The moments of the $S N T-B S$ distribution depend on the moments of the $S N T$ distribution, but they can also be expressed in terms of the moments of the $B S$ distribution. In the following proposition, we present a relationship between the means, variances, coefficients of variation $(C V)$, skewness $(C S)$ and kurtosis $(C K)$ of the $S N T-B S$ and $B S$ distributions.

Proposition 3. Let $T_{S N T} \sim S N T-B S(\alpha, \beta, \lambda, \nu)$ and $T_{N} \sim B S(\alpha, \beta)$. Then the mean, variance, $\operatorname{CV}(\gamma[T]), \operatorname{CS}\left(\alpha_{3}[T]\right)$, and $\operatorname{CK}\left(\alpha_{4}[T]\right)$ of these two random variables satisfy the following:

$$
\begin{aligned}
& \text { (i) } \quad E\left[T_{S N T}\right]=E\left(T_{N}\right)+\frac{\alpha \beta}{2} \omega_{1} \\
& \text { (ii) } \quad \operatorname{Var}\left[T_{S N T}\right]=\operatorname{Var}\left(T_{N}\right)+\left[\frac{\alpha \beta}{2}\right] \alpha_{\omega} \\
& \text { (iii) } \gamma\left[T_{S N T}\right]=\gamma\left[T_{N}\right] \frac{\sqrt{1+[\alpha \beta]^{2} \alpha_{\omega} / \operatorname{Var}\left[2 T_{N}\right]}}{1+\alpha \beta \omega_{1} / E\left[2 T_{N}\right]} \\
& \text { (iv) } \alpha_{3}\left[T_{S N T}\right]=\alpha_{3}\left[T_{N}\right]\left[\frac{4+5 \alpha^{2}}{4+5 \alpha^{2}+\alpha_{\omega}}\right]^{\frac{3}{2}}+\frac{2\left[a_{0}+a_{1} \alpha+a_{2} \alpha^{2}\right]}{\left[4+5 \alpha^{2}+\alpha_{\omega}\right]^{\frac{3}{2}}} \\
& \text { (v) } \alpha_{4}\left[T_{S N T}\right]=\left[\alpha_{4}\left[T_{N}\right]+\frac{b_{0}+b_{1} \alpha+b_{2} \alpha^{2}+b_{3} \alpha^{3}}{\left[4+5 \alpha^{2}\right]^{2}}\right] \frac{\left[4+5 \alpha^{2}\right]^{2}}{\left[4+5 \alpha^{2}+\alpha_{\omega}\right]^{2}}
\end{aligned}
$$

where

$$
\begin{aligned}
\alpha_{\omega} & =2 \alpha\left[\omega_{3}-\omega_{1}\right]-\omega_{1}^{2}, \\
a_{0} & =2 \omega_{3}-6 \omega_{1}+\omega_{1}^{3}, \\
a_{1} & =3 \omega_{1}^{2}-3 \omega_{1} \omega_{3}, \\
a_{2} & =2 \omega_{5}-6 \omega_{1}-3 \omega_{3}, \\
b_{0} & =24 \omega_{1}^{2}-3 \omega_{1}^{4}-16 \omega_{1} \omega_{3}, \\
b_{1} & =16 \omega_{5}+12 \omega_{1}^{2} \omega_{3}-96 \omega_{1}-12 \omega_{1}^{3}-16 \omega_{3}, \\
b_{2} & =18 \omega_{1}^{5}+24 \omega_{1} \omega_{3}-16 \omega_{1} \omega_{5}, \\
b_{3} & =8 \omega_{7}-168 \omega_{1}-16 \omega_{5},
\end{aligned}
$$

and $\omega_{k}=E\left[Z^{k} \sqrt{\alpha^{2} Z^{2}+4}\right], Z \sim S N T(\lambda, \nu)$ and $k=1,3,5,7$. In order to calculate values for $\omega_{k}$, the integrals involved must be solved using numerical methods. 
From (1) and (6), we readily obtain the stochastic representation

$$
\begin{aligned}
T \stackrel{d}{=} & \frac{\beta}{4}\left[\alpha\left\{\frac{Y_{1}}{\sqrt{1+\lambda^{2} \tau}}+\lambda \sqrt{\frac{\tau}{1+\lambda^{2} \tau}}\left|Y_{0}\right|\right\}\right. \\
& \left.+\sqrt{\left\{\alpha\left(\frac{Y_{1}}{\sqrt{1+\lambda^{2} \tau}}+\lambda \sqrt{\frac{\tau}{1+\lambda^{2} \tau}}\left|Y_{0}\right|\right)\right\}^{2}+4}\right]^{2}
\end{aligned}
$$

where now $T \sim S N T-B S(\alpha, \beta, \lambda, \nu)$. Thus,

$$
Z^{2}=\frac{1}{\alpha^{2}}\left[\frac{T}{\beta}+\frac{\beta}{T}-2\right] \sim \chi^{2}(1) .
$$

and hence

$$
Z \stackrel{d}{=} \frac{1}{\alpha}\left[\sqrt{\frac{T}{\beta}}-\sqrt{\frac{\beta}{T}}\right] \sim S N T(\lambda, \nu) .
$$

Putting $\gamma=\sqrt{\frac{1+\lambda^{2} \tau}{\tau}}\left|Y_{0}\right|$, a further hierarchical representation of the $S N T-$ $B S$ distribution can be written as

$$
\begin{aligned}
T \mid \gamma, \tau & \sim E B S\left(\frac{\alpha}{\sqrt{1+\lambda^{2} \tau}}, \beta, 2,-\frac{\lambda \gamma \tau}{\sqrt{1+\lambda^{2} \tau}}\right), \\
\gamma \mid \tau & \sim T N\left(0, \frac{1+\lambda^{2} \tau}{\tau} ;(0, \infty)\right), \\
\tau & \sim \Gamma(\nu / 2, \nu / 2),
\end{aligned}
$$

where $E B S(\alpha, \beta, \sigma, \lambda)$ denotes the extended $B S$ distribution introducted by Leiva et al. (2010) and $T N\left(\mu, \sigma^{2} ;(a, b)\right)$ denotes the truncated normal distribution for $N\left(\mu, \sigma^{2}\right)$ lying within the interval $(a, b)$.

From (11), the joint pdf of $T, \gamma$ and $\tau$ is given by

$$
\begin{aligned}
f(t, \gamma, \tau) & =\frac{1}{\pi} \cdot \frac{\left(\frac{\nu}{2}\right)^{\frac{\nu}{2}}}{\Gamma\left(\frac{\nu}{2}\right)} \tau^{\frac{\nu+1}{2-1}} \exp \left\{-\frac{\nu \tau}{2}\right\} I(\gamma>0) \\
& \times \exp \left\{-\frac{1}{2} a^{2}(t ; \alpha, \beta)-\frac{1}{2} \tau(\gamma-\lambda a(t ; \alpha, \beta))^{2}\right\} A(t ; \alpha, \beta) .
\end{aligned}
$$


Integrating out $\gamma$ in (12), we get

$$
\begin{aligned}
f(t, \tau)= & \sqrt{\frac{2}{\pi}} \frac{\left(\frac{\nu}{2}\right)^{\frac{\nu}{2}}}{\Gamma\left(\frac{\nu}{2}\right)} \tau^{\frac{\nu}{2}-1} \exp \left\{-\frac{a^{2}(t ; \alpha, \beta)+\nu \tau}{2}\right\} \\
& \times \Phi(\lambda \sqrt{\tau} a(t ; \alpha, \beta)) A(t ; \alpha, \beta) .
\end{aligned}
$$

Dividing (12) by (13) gives

$$
f(\gamma \mid \tau, t)=\frac{1}{\Phi(\lambda \sqrt{\tau} a(t ; \alpha, \beta))} \sqrt{\frac{\tau}{2 \pi}} \times \exp \left\{-\frac{1}{2} \tau(\gamma-\lambda a(t ; \alpha, \beta))\right\} .
$$

It follows from (14) that the conditional distribution of $\gamma$ given $\tau$ and $T$ is

$$
\gamma \mid \tau, t \sim T N\left(\lambda a(t ; \alpha, \beta), \tau^{-1} ;(0, \infty)\right) .
$$

Moreover, dividing (13) by (7) yields

$$
f(\tau \mid t)=\frac{\left(\frac{\nu}{2}\right)^{\frac{\nu}{2}}}{\Gamma\left(\frac{\nu}{2}\right)} \tau^{\frac{\nu}{2-1}} \exp \left\{-\frac{\nu \tau}{2}\right\} \frac{\Phi(\lambda \sqrt{\tau} a(t ; \alpha, \beta))}{T(\lambda a(t ; \alpha, \beta) ; \nu)} .
$$

It follows from (16) that the conditional distribution of $\tau$ given $T=t$ is gamma-weighted.

Proposition 4. Given the hierarchical representation (11), the following hold:

(a) The conditional expectation of $\tau$ given $T=t$ is

$$
E(\tau \mid T=t)=\frac{T\left(\lambda a(t ; \alpha, \beta) \sqrt{\frac{2}{\nu}+1} ; \nu+2\right)}{T(\lambda a(t ; \alpha, \beta) ; \nu)} .
$$

(b) The conditional expectation of $\frac{\phi(\sqrt{\tau} \lambda a(t ; \alpha, \beta))}{\Phi(\sqrt{\tau} \lambda a(t ; \alpha, \beta))} \sqrt{\tau}$ given $T=t$ is

$$
\begin{aligned}
E\left(\frac{\phi(\sqrt{\tau} \lambda a(t ; \alpha, \beta))}{\Phi(\sqrt{\tau} \lambda a(t ; \alpha, \beta))}\right. & \sqrt{\tau} \mid T=t)= \\
& \frac{\Gamma\left(\frac{\nu+1}{2}\right)\left(\frac{\nu}{2}\right)^{\frac{\nu}{2}}}{\sqrt{2 \pi} \Gamma\left(\frac{\nu}{2}\right) T(\lambda a(t ; \alpha, \beta) ; \nu)\left(\frac{\nu+\lambda^{2} a^{2}(t ; \alpha, \beta)}{2}\right)^{\frac{\nu+1}{2}}} .
\end{aligned}
$$


(c) The conditional expectation of $\gamma \tau$ given $T=t$ is

$$
\begin{aligned}
E(\gamma \tau \mid T=t)= & \lambda a(t ; \alpha, \beta) E(\tau \mid T=t) \\
& +E\left(\frac{\phi(\sqrt{\tau} \lambda a(t ; \alpha, \beta))}{\Phi(\sqrt{\tau} \lambda a(t ; \alpha, \beta))} \sqrt{\tau} \mid T=t\right) .
\end{aligned}
$$

(d) The conditional expectation of $\gamma^{2} \tau$ given $T=t$ is

$$
\begin{aligned}
E\left(\gamma^{2} \tau \mid T=t\right)= & \lambda^{2} a^{2}(t ; \alpha, \beta) E(\tau \mid T=t)+1 \\
& +\lambda a(t ; \alpha, \beta) E\left(\frac{\phi(\sqrt{\tau} \lambda a(t ; \alpha, \beta))}{\Phi(\sqrt{\tau} \lambda a(t ; \alpha, \beta))} \sqrt{\tau} \mid T=t\right) .
\end{aligned}
$$

(e) The conditional expectation of $\log (\tau)$ given $T=t$ is

$$
\begin{aligned}
E(\log (\tau) \mid T=t)= & D G\left(\frac{\nu+1}{2}\right)-\log \frac{\nu}{2}-1+E(\tau \mid T=t)-\frac{1}{\nu} \\
& +\frac{1}{T(\lambda a(t ; \alpha, \beta) ; \nu)} \int_{-\infty}^{\lambda a(t ; \alpha, \beta)} g_{\nu}(x) t(x ; \nu) d x,
\end{aligned}
$$

where

$$
g_{\nu}(x)=\frac{(\nu+1) x^{2}}{\nu\left(1+x^{2}\right)}-\log \left(1+\frac{\nu}{2}\right)
$$

$t(. ; \nu)$ the pdf of Student's t distribution and $D G(x)=\frac{\Gamma^{\prime}(x)}{\Gamma(x)}$ is the digamma function.

Proof. See Appendix B.

\section{Maximum Likelihood Estimation}

The EM algorithm is a widely applicable approach for iterative computation of the ML estimates, and is useful in a variety of incomplete data problems. Each iteration of the EM-algorithm, consists of two steps: the Expectation step or the E-step and the Maximization step or the M-step. In this section, we discuss the ML estimation of the $S N T-B S$ using the ECM algorithm, which is a modification of the usual EM algorithm. For more details about the EM and ECM algorithms, we refer to Dempster et al. (1977) and Meng and Rubin (1993), respectively. 


\subsection{Estimation Via the ECM Algorithm}

From the representation (11), $n$ independent observations, each following $S N T-B S(\alpha, \beta, \lambda, \nu)$, can be expressed as

$$
\begin{aligned}
T_{i} \mid \gamma_{i}, \tau_{i} & \stackrel{i n d}{\sim} \operatorname{EBS}\left(\frac{\alpha}{\sqrt{1+\lambda^{2} \tau_{i}}}, \beta, 2,-\frac{\lambda \gamma_{i} \tau_{i}}{\sqrt{1+\lambda^{2} \tau_{i}}}\right), \\
\gamma_{i} \mid \tau_{i} & \stackrel{i n d}{\sim} T N\left(0, \frac{1+\lambda^{2} \tau_{i}}{\tau_{i}} ;(0, \infty)\right), \\
\tau_{i} & \stackrel{\text { ind }}{\sim} \Gamma\left(\frac{\nu}{2}, \frac{\nu}{2}\right), \quad i=1, \ldots, n .
\end{aligned}
$$

Letting $\boldsymbol{t}=\left(t_{1}, \ldots, t_{n}\right), \boldsymbol{\gamma}=\left(\gamma_{1}, \ldots, \gamma_{n}\right)$ and $\boldsymbol{\tau}=\left(\tau_{1}, \ldots, \tau_{n}\right)$, the complete data $\log$-likelihood function of $\boldsymbol{\theta}=(\alpha, \beta, \lambda, \nu)$ given $\mathbf{v}=(\boldsymbol{t}, \boldsymbol{\gamma}, \boldsymbol{\tau})$, aside from additive constants, is

$$
\begin{aligned}
\ell_{c}(\boldsymbol{\theta} ; \mathbf{v})= & \frac{n \nu}{2} \log \left(\frac{\nu}{2}\right)-n \log \Gamma\left(\frac{\nu}{2}\right)-n \log \alpha+\frac{\nu-1}{2} \sum_{i=1}^{n} \log \tau_{i}-\frac{1}{2} \sum_{i=1}^{n} \tau_{i} \gamma_{i}^{2} \\
& +\sum_{i=1}^{n} \log \left(\frac{t_{i}+\beta}{\sqrt{\beta}}\right)-\frac{\nu}{2} \sum_{i=1}^{n} \tau_{i}-\frac{1}{2 \alpha^{2}} \sum_{i=1}^{n}\left[1+\lambda^{2} \tau_{i}\right] \xi^{2}\left(t_{i} ; \beta\right) \\
& +\frac{\lambda}{\alpha} \sum_{i=1}^{n} \tau_{i} \gamma_{i} \xi\left(t_{i} ; \beta\right),
\end{aligned}
$$

where $\xi\left(t_{i} ; \beta\right)=\sqrt{\frac{t_{i}}{\beta}}-\sqrt{\frac{\beta}{t_{i}}}$.

Suppose $\hat{\boldsymbol{\theta}}^{(k)}=\left(\hat{\alpha}^{(k)}, \hat{\beta}^{(k)}, \hat{\lambda}^{(k)}, \hat{\nu}^{(k)}\right)$ is the current estimate (in the $k$ th iteration) of $\boldsymbol{\theta}$. Based on the EM algorithm principle, in the E-step, we need to calculate the conditional expectation $Q\left(\boldsymbol{\theta} \mid \hat{\boldsymbol{\theta}}^{(k)}\right)=E\left(\ell_{c}(\boldsymbol{\theta} ; \mathbf{v}) \mid \mathbf{t}\right)$. After some algebra we obtain

$$
\begin{aligned}
Q\left(\boldsymbol{\theta} \mid \hat{\boldsymbol{\theta}}^{(k)}\right)= & \frac{n \nu}{2} \log \left(\frac{\nu}{2}\right)-n \log \Gamma\left(\frac{\nu}{2}\right)-n \log \alpha+\frac{\nu-1}{2} \sum_{i=1}^{n} \hat{s}_{4 i}^{(k)}-\frac{1}{2} \sum_{i=1}^{n} \hat{s}_{3 i}^{(k)} \\
& +\sum_{i=1}^{n} \log \left(\frac{t_{i}+\beta}{\sqrt{\beta}}\right)-\frac{\nu}{2} \sum_{i=1}^{n} \hat{s}_{1 i}^{(k)}-\frac{1}{2 \alpha^{2}} \sum_{i=1}^{n}\left[1+\lambda^{2} \hat{s}_{1 i}^{(k)}\right] \xi^{2}\left(t_{i} ; \beta\right) \\
& +\frac{\lambda}{\alpha} \sum_{i=1}^{n} \hat{s}_{2 i}^{(k)} \xi\left(t_{i} ; \beta\right),
\end{aligned}
$$


where

$$
\begin{aligned}
\hat{s}_{1 i}^{(k)}= & E\left(\tau_{i} \mid t_{i}, \hat{\boldsymbol{\theta}}^{(k)}\right)=\frac{T\left(\hat{\lambda}^{(k)} a\left(t_{i} ; \hat{\alpha}^{(k)}, \hat{\beta}^{(k)}\right) \sqrt{\frac{2}{\hat{\nu}^{(k)}}+1} ; \hat{\nu}^{(k)}+2\right)}{T\left(\hat{\lambda}^{(k)} a\left(t_{i} ; \hat{\alpha}^{(k)}, \hat{\beta}^{(k)}\right) ; \hat{\nu}^{(k)}\right)}, \\
\hat{s}_{2 i}^{(k)}= & E\left(\tau_{i} \gamma_{i} \mid t_{i}, \hat{\boldsymbol{\theta}}^{(k)}\right)=\hat{\lambda}^{(k)} a\left(t_{i} ; \hat{\alpha}^{(k)}, \hat{\beta}^{(k)}\right) \hat{s}_{1 i}^{(k)}+\hat{s}_{5 i}^{(k)}, \\
\hat{s}_{3 i}^{(k)}= & E\left(\tau_{i} \gamma_{i}^{2} \mid t_{i}, \hat{\boldsymbol{\theta}}^{(k)}\right)=\hat{\lambda}^{2^{(k)}} a^{2}\left(t_{i} ; \hat{\alpha}^{(k)}, \hat{\beta}^{(k)}\right) \hat{s}_{1 i}^{(k)}+1 \\
& +\hat{\lambda}^{(k)} a\left(t_{i} ; \hat{\alpha}^{(k)}, \hat{\beta}^{(k)}\right) \hat{s}_{5 i}^{(k)},
\end{aligned}
$$

and

$$
\begin{aligned}
\hat{s}_{4 i}^{(k)} & =E\left(\log \tau_{i} \mid t_{i}, \hat{\boldsymbol{\theta}}^{(k)}\right) \\
& =D G\left(\frac{\hat{\nu}^{(k)}+1}{2}\right)-\log \frac{\hat{\nu}^{(k)}}{2}-1+\hat{s}_{1 i}^{(k)}-\frac{1}{\hat{\nu}^{(k)}} \\
& +\frac{1}{T\left(\hat{\lambda}^{(k)} a\left(t_{i} ; \hat{\alpha}^{(k)}, \hat{\beta}^{(k)}\right) ; \hat{\nu}^{(k)}\right)} \int_{-\infty}^{\hat{\lambda}^{(k)} a\left(t_{i} ; \hat{\alpha}^{(k)}, \hat{\beta}^{(k)}\right)} g_{\hat{\nu}^{(k)}}(x) t\left(x ; \hat{\nu}^{(k)}\right) d x,
\end{aligned}
$$

with

$$
\begin{aligned}
\hat{s}_{5 i}^{(k)}= & E\left(\frac{\phi\left(\sqrt{\tau_{i}} \lambda a\left(t_{i} ; \alpha, \beta\right)\right)}{\Phi\left(\sqrt{\tau_{i}} \lambda a\left(t_{i} ; \alpha, \beta\right)\right)} \sqrt{\tau_{i}} \mid t_{i}, \hat{\boldsymbol{\theta}}^{(k)}\right) \\
= & \frac{\Gamma\left(\frac{\hat{\nu}^{(k)}+1}{2}\right)\left(\frac{\hat{\nu}^{(k)}}{2}\right)^{\frac{\hat{\nu}^{(k)}}{2}}}{\sqrt{2 \pi} \Gamma\left(\frac{\hat{\nu}^{(k)}}{2}\right) T\left(\hat{\lambda}^{(k)} a\left(t ; \hat{\alpha}^{(k)}, \hat{\beta}^{(k)}\right) ; \hat{\nu}^{(k)}\right)\left(\frac{\hat{\nu}^{(k)}+\hat{\lambda}^{2(k)} a^{2}\left(t ; \hat{\alpha}^{(k)}, \hat{\beta}^{(k)}\right)}{2}\right)^{\frac{\hat{\nu}^{(k)}+1}{2}}} .
\end{aligned}
$$

We then have the following ECM algorithm:

E-step: Given $\boldsymbol{\theta}=\hat{\boldsymbol{\theta}}^{(k)}$, compute $\hat{s}_{1 i}^{(k)}, \hat{s}_{2 i}^{(k)}, \hat{s}_{3 i}^{(k)}, \hat{s}_{4 i}^{(k)}$ and $\hat{s}_{5 i}^{(k)}$ from Equations (17)-(21) for $j=1, \ldots, n$. 
CM-step 1: Update $\hat{\alpha}^{2^{(k)}}$ by maximizing (17) over $\alpha^{2}$ which leads to

$$
\hat{\alpha}^{2^{(k+1)}}=\frac{1}{n} \sum_{i=1}^{n} \xi^{2}\left(t_{i} ; \hat{\beta}^{(k)}\right) .
$$

CM-step 2: Fix $\alpha=\hat{\alpha}^{(k+1)}$, update $\hat{\lambda}^{(k)}$ by maximizing (17) over $\lambda$, which gives

$$
\hat{\lambda}^{(k+1)}=\hat{\alpha}^{(k+1)} \frac{\sum_{i=1}^{n} \hat{s}_{2 i}^{(k)} \xi\left(t_{i} ; \hat{\beta}^{(k)}\right)}{\sum_{i=1}^{n} \hat{s}_{1 i}^{(k)} \xi^{2}\left(t_{i} ; \hat{\beta}^{(k)}\right)} .
$$

CM-step 3: Fix $\alpha=\hat{\alpha}^{(k+1)}, \lambda=\hat{\lambda}^{(k+1)}$ obtain $\hat{\nu}^{(k+1)}$ as the solution of

$$
\frac{n}{2}\left(\log \left(\frac{\nu}{2}\right)+1\right)-\frac{n}{2} D G\left(\frac{\nu}{2}\right)-\frac{1}{2} \sum_{i=1}^{n} \hat{s}_{1 i}^{(k)}+\frac{1}{2} \sum_{i=1}^{n} \hat{s}_{4 i}^{(k)}=0 .
$$

CM-step 4: Fix $\alpha=\hat{\alpha}^{(k+1)}, \lambda=\hat{\lambda}^{(k+1)}$ and $\nu=\hat{\nu}^{(k+1)}$, obtain $\hat{\beta}^{(k+1)}$ using

$$
\hat{\beta}^{(k+1)}=\underset{\beta}{\arg \max } Q\left(\hat{\alpha}^{(k+1)}, \beta, \hat{\lambda}^{(k+1)}, \hat{\nu}^{(k+1)} \mid \hat{\boldsymbol{\theta}}^{(k+1)}\right) .
$$

Note that the CM-Steps 3 and 4 respectively require an one-dimensional search for the root of $\nu$ and optimization with respect to $\beta$, and can be easily carried out using the uniroot and optimize function built in the statistical software $R$.

\section{Remark 1.}

(i) The ECM algorithm has several appealing features, such as stability of monotone convergence with each iteration and increase in the likelihood at each iteration. It is also not sensitivity to initial values. However, the convergence rate may be too slow for practical use. To accelerate the convergence, we combine the ECM and Newton-Raphson (Numerical) methods using estimates obtained from the Newton-Raphson method as the initial point of ECM method. The key feature of the hybrid procedure is that it combines the stability of the ECM algorithm with the rapid convergence of the Newton-Raphson method, yielding a substantial improvement in computational efficiency. 
(ii) Useful starting values for implementing the ECM algorithm can be easily obtained from an $R$ package called $b s$, on $C R A N$.R-project.org; see Leiva et al. (2006). This procedure is based on the modified moment estimators given by $\hat{\alpha}_{0}=\left(2(s / r)^{1 / 2}-1\right)^{1 / 2}$ and $\hat{\beta}_{0}=(s r)^{1 / 2}$, where

$$
s=\frac{1}{n} \sum_{i=1}^{n} t_{i}, \quad \text { and } \quad r=\frac{1}{\frac{1}{n} \sum_{i=1}^{n} \frac{1}{t_{i}}} .
$$

We now use (10) to obtain a sample of $n$ observations from a $S N T$ distribution

$$
z_{i}=\frac{1}{\hat{\alpha}_{0}}\left[\sqrt{\frac{t_{i}}{\hat{\beta}_{0}}}-\sqrt{\frac{\hat{\beta}_{0}}{t_{i}}}\right], \quad i=1, \ldots, n
$$

The initial estimates $\hat{\lambda}_{0}$ and $\hat{\nu}_{0}$ are obtained from moments using numerical methods such as, the procedure DEoptim in the statistical software $R$ for maximizing the corresponding likelihood function.

(iii) To ensure that true ML estimates are obtained, we recommend running the ECM algorithm using a range of different starting values and checking whether all of them result in the same estimates.

\subsection{Evaluation of the Standard Errors from the EM-Algorithm}

In this section, we use the results of Louis (1982) to obtain the standard errors of estimators by the EM-algorithm. Let $\mathbf{v}=(\boldsymbol{t}, \boldsymbol{\gamma}, \boldsymbol{\tau})$, then the elements of 
the $4 \times 4$ observed information matrix $I(\boldsymbol{\theta} ; \mathbf{v})=-\left[\frac{\partial^{2} \ell_{c}(\boldsymbol{\theta} ; \mathbf{v})}{\partial \boldsymbol{\theta}^{2}}\right]$ are given by

$$
\begin{aligned}
\frac{\partial^{2} \ell_{c}(\boldsymbol{\theta} ; \mathbf{v})}{\partial \alpha^{2}} & =-\frac{3}{\alpha^{4}} \sum_{i=1}^{n} \xi^{2}\left(t_{i} ; \beta\right)+\frac{2 \lambda}{\alpha^{3}} \sum_{i=1}^{n} \tau_{i} \gamma_{i} \xi\left(t_{i} ; \beta\right)-\frac{3 \lambda^{2}}{\alpha^{4}} \sum_{i=1}^{n} \tau_{i} \xi^{2}\left(t_{i} ; \beta\right)+\frac{n}{\alpha^{2}}, \\
\frac{\partial^{2} \ell_{c}(\boldsymbol{\theta} ; \mathbf{v})}{\partial \alpha \partial \beta} & =\frac{2}{\alpha^{3}} \sum_{i=1}^{n} \xi\left(t_{i} ; \beta\right) \xi^{\prime}\left(t_{i} ; \beta\right)-\frac{\lambda}{\alpha^{2}} \sum_{i=1}^{n} \tau_{i} \gamma_{i} \xi^{\prime}\left(t_{i} ; \beta\right), \\
\frac{\partial^{2} \ell_{c}(\boldsymbol{\theta} ; \mathbf{v})}{\partial \alpha \partial \lambda} & =-\frac{1}{\alpha^{2}} \sum_{i=1}^{n} \tau_{i} \gamma_{i} \xi\left(t_{i} ; \beta\right)+\frac{2 \lambda}{\alpha^{3}} \sum_{i=1}^{n} \tau_{i} \xi^{2}\left(t_{i} ; \beta\right), \\
\frac{\partial^{2} \ell_{c}(\boldsymbol{\theta} ; \mathbf{v})}{\partial \beta^{2}} & =\frac{1}{\alpha^{2}} \sum_{i=1}^{n} \xi^{\prime 2}\left(t_{i} ; \beta\right)-\frac{1}{\alpha^{2}} \sum_{i=1}^{n} \xi^{\prime \prime}\left(t_{i} ; \beta\right) \xi\left(t_{i} ; \beta\right)+\frac{\lambda}{\alpha} \sum_{i=1}^{n} \tau_{i} \gamma_{i} \xi^{\prime \prime}\left(t_{i} ; \beta\right) \\
& -\frac{\lambda^{2}}{\alpha^{2}} \sum_{i=1}^{n} \tau_{i} \xi^{\prime 2}\left(t_{i} ; \beta\right)-\frac{\lambda^{2}}{\alpha^{2}} \sum_{i=1}^{n} \tau_{i} \xi^{\prime \prime}\left(t_{i} ; \beta\right) \xi\left(t_{i} ; \beta\right) \\
& -\sum_{i=1}^{n} \frac{1}{\left(t_{i}+\beta\right)^{2}}+\frac{n}{2 \beta^{2}},
\end{aligned}
$$

$$
\begin{aligned}
& \frac{\partial^{2} \ell_{c}(\boldsymbol{\theta} ; \mathbf{v})}{\partial \beta \partial \lambda}=\frac{1}{\alpha} \sum_{i=1}^{n} \tau_{i} \gamma_{i} \xi^{\prime}\left(t_{i} ; \beta\right)-\frac{2 \lambda}{\alpha^{2}} \sum_{i=1}^{n} \tau_{i} \xi\left(t_{i} ; \beta\right) \xi^{\prime}\left(t_{i} ; \beta\right) \\
& \frac{\partial^{2} \ell_{c}(\boldsymbol{\theta} ; \mathbf{v})}{\partial \lambda^{2}}=-\frac{1}{\alpha^{2}} \sum_{i=1}^{n} \tau_{i} \xi^{2}\left(t_{i} ; \beta\right) \\
& \frac{\partial^{2} \ell_{c}(\boldsymbol{\theta} ; \mathbf{v})}{\partial \nu^{2}}=\frac{2}{2 \nu}-\frac{n}{2} \frac{\partial D G\left(\frac{\nu}{2}\right)}{\partial \nu} \\
& \frac{\partial^{2} \ell_{c}(\boldsymbol{\theta} ; \mathbf{v})}{\partial \alpha \partial \nu}=\frac{\partial^{2} \ell_{c}(\boldsymbol{\theta} ; \mathbf{v})}{\partial \beta \partial \nu}=\frac{\partial^{2} \ell_{c}(\boldsymbol{\theta} ; \mathbf{v})}{\partial \lambda \partial \nu}=0
\end{aligned}
$$

where $\xi^{\prime}\left(t_{i} ; \beta\right)=\frac{\partial \xi\left(t_{i} ; \beta\right)}{\partial \beta}=-\frac{\beta+t}{2 \sqrt{t} \sqrt{\beta^{3}}}$ and $\xi^{\prime \prime}\left(t_{i} ; \beta\right)=\frac{\partial^{2} \xi\left(t_{i} ; \beta\right)}{\partial \beta^{2}}=\frac{\beta+3 t}{4 \sqrt{t} \sqrt{\beta^{5}}}$.

Taking the conditional expectation of $I(\boldsymbol{\theta} ; \mathbf{V})$ given $\mathbf{t}$, we obtain the $4 \times 4$ matrix

$$
\ell_{c}^{*}(\boldsymbol{\theta} ; \boldsymbol{t})=E(I(\boldsymbol{\theta} ; \mathbf{V}) \mid \boldsymbol{t})=\left[c_{i j}\right]
$$


where

$$
\begin{aligned}
c_{11}= & -\frac{3}{\alpha^{4}} \sum_{i=1}^{n} \xi^{2}\left(t_{i} ; \beta\right)+\frac{2 \lambda}{\alpha^{3}} \sum_{i=1}^{n} s_{2 i} \xi\left(t_{i} ; \beta\right)-\frac{3 \lambda^{2}}{\alpha^{4}} \sum_{i=1}^{n} s_{1 i} \xi^{2}\left(t_{i} ; \beta\right)+\frac{n}{\alpha^{2}} \\
c_{12}= & c_{21}=\frac{2}{\alpha^{3}} \sum_{i=1}^{n} \xi\left(t_{i} ; \beta\right) \xi^{\prime}\left(t_{i} ; \beta\right)-\frac{\lambda}{\alpha^{2}} \sum_{i=1}^{n} s_{2 i} \xi^{\prime}\left(t_{i} ; \beta\right), \\
c_{13}= & c_{31}=-\frac{1}{\alpha^{2}} \sum_{i=1}^{n} s_{2 i} \xi\left(t_{i} ; \beta\right)+\frac{2 \lambda}{\alpha^{3}} \sum_{i=1}^{n} s_{1 i} \xi^{2}\left(t_{i} ; \beta\right), \\
c_{22}= & \frac{1}{\alpha^{2}} \sum_{i=1}^{n} \xi^{\prime 2}\left(t_{i} ; \beta\right)-\frac{1}{\alpha^{2}} \sum_{i=1}^{n} \xi^{\prime \prime}\left(t_{i} ; \beta\right) \xi\left(t_{i} ; \beta\right)+\frac{\lambda}{\alpha} \sum_{i=1}^{n} s_{2 i} \xi^{\prime \prime}\left(t_{i} ; \beta\right) \\
& -\frac{\lambda^{2}}{\alpha^{2}} \sum_{i=1}^{n} s_{1 i} \xi^{2}\left(t_{i} ; \beta\right)-\frac{\lambda^{2}}{\alpha^{2}} \sum_{i=1}^{n} s_{1 i} \xi^{\prime \prime}\left(t_{i} ; \beta\right) \xi\left(t_{i} ; \beta\right) \\
& -\sum_{i=1}^{n} \frac{1}{\left(t_{i}+\beta\right)^{2}}+\frac{n}{2 \beta^{2}}, \\
c_{23}= & c_{32}=\frac{1}{\alpha} \sum_{i=1}^{n} s_{2 i} \xi^{\prime}\left(t_{i} ; \beta\right)-\frac{2 \lambda}{\alpha^{2}} \sum_{i=1}^{n} s_{1 i} \xi\left(t_{i} ; \beta\right) \xi^{\prime}\left(t_{i} ; \beta\right), \\
c_{33}= & -\frac{1}{\alpha^{2}} \sum_{i=1}^{n} s_{2 i} \xi^{2}\left(t_{i} ; \beta\right), \\
c_{44}= & \frac{2}{2 \nu}-\frac{n}{2} \frac{\partial D G\left(\frac{\nu}{2}\right)}{\partial \nu}, \\
c_{14}= & c_{41}=c_{24}=c_{42}=c_{34}=c_{43}=0
\end{aligned}
$$

with

$$
s_{1 i}=\frac{T\left(\lambda a(t ; \alpha, \beta) \sqrt{\frac{2}{\nu}+1} ; \nu+2\right)}{T(\lambda a(t ; \alpha, \beta) ; \nu)}, \quad s_{2 i}=\lambda a(t ; \alpha, \beta) s_{1 i}+s_{5 i},
$$

and

$$
s_{5 i}=\frac{\Gamma\left(\frac{\nu+1}{2}\right)\left(\frac{\nu}{2}\right)^{\frac{\nu}{2}}}{\sqrt{2 \pi} T(\lambda a(t ; \alpha, \beta) ; \nu)\left(\frac{\nu+\lambda^{2} a^{2}(t ; \alpha, \beta)}{2}\right)^{\frac{\nu+1}{2}}} .
$$

Moving now to the computation of $\ell_{m}^{*}(\boldsymbol{\theta} ; \boldsymbol{t})$ we have

$$
\ell_{m}^{*}(\boldsymbol{\theta} ; \boldsymbol{t})=\operatorname{Var}\left(\frac{\partial \ell_{c}(\boldsymbol{\theta} ; \mathbf{V})}{\partial \boldsymbol{\theta}} \mid \boldsymbol{t}\right)=\left[v_{i j}\right],
$$


where

$$
\begin{aligned}
& v_{11}=\sum_{i=1}^{n}\left[\frac{\lambda^{4}}{\alpha^{6}} \xi^{4}\left(t_{i} ; \beta\right) \mathcal{C}_{1 i}+\frac{\lambda^{2}}{\alpha^{4}} \xi^{2}\left(t_{i} ; \beta\right) \mathcal{C}_{2 i}-2 \frac{\lambda^{3}}{\alpha^{5}} \xi^{3}\left(t_{i} ; \beta\right) \mathcal{C}_{3 i}\right], \\
& v_{12}=v_{21}=\sum_{i=1}^{n}\left[-\frac{\lambda^{4}}{\alpha^{5}} \xi^{3}\left(t_{i} ; \beta\right) \xi^{\prime}\left(t_{i} ; \beta\right) \mathcal{C}_{1 i}-\frac{\lambda^{2}}{\alpha^{3}} \xi\left(t_{i} ; \beta\right) \xi^{\prime}\left(t_{i} ; \beta\right) \mathcal{C}_{2 i}\right. \\
& \left.+2 \frac{\lambda^{3}}{\alpha^{4}} \xi^{2}\left(t_{i} ; \beta\right) \xi^{\prime}\left(t_{i} ; \beta\right) \mathcal{C}_{3 i}\right] \\
& v_{13}=v_{31}=\sum_{i=1}^{n}\left[-\frac{\lambda^{3}}{\alpha^{5}} \xi^{4}\left(t_{i} ; \beta\right) \mathcal{C}_{1 i}-\frac{\lambda}{\alpha^{3}} \xi^{2}\left(t_{i} ; \beta\right) \mathcal{C}_{2 i}+\frac{\lambda^{2}}{\alpha^{4}} \xi^{3}\left(t_{i} ; \beta\right) \mathcal{C}_{3 i}\right], \\
& v_{14}=v_{41}=\sum_{i=1}^{n}\left[\frac{\lambda}{2 \alpha^{2}} \xi^{2}\left(t_{i} ; \beta\right) \mathcal{C}_{4 i}+\frac{\lambda^{2}}{2 \alpha^{3}} \xi^{2}\left(t_{i} ; \beta\right) \mathcal{C}_{5 i}-\frac{\lambda^{2}}{2 \alpha^{3}} \xi^{2}\left(t_{i} ; \beta\right) \mathcal{C}_{1 i}\right] \text {, } \\
& v_{22}=\sum_{i=1}^{n}\left[\frac{\lambda^{4}}{\alpha^{4}} \xi^{2}\left(t_{i} ; \beta\right) \xi^{\prime 2}\left(t_{i} ; \beta\right) \mathcal{C}_{1 i}-\frac{\lambda^{2}}{\alpha^{2}} \xi^{\prime 2}\left(t_{i} ; \beta\right) \mathcal{C}_{2 i}-2 \frac{\lambda^{3}}{\alpha^{3}} \xi\left(t_{i} ; \beta\right) \xi^{\prime 2}\left(t_{i} ; \beta\right) \mathcal{C}_{3 i}\right] \text {, } \\
& v_{23}=v_{32}=\sum_{i=1}^{n}\left[\frac{\lambda^{3}}{\alpha^{4}} \xi^{3}\left(t_{i} ; \beta\right) \xi^{\prime}\left(t_{i} ; \beta\right) \mathcal{C}_{1 i}+\frac{\lambda}{\alpha^{2}} \xi\left(t_{i} ; \beta\right) \xi^{\prime}\left(t_{i} ; \beta\right) \mathcal{C}_{2 i}\right. \\
& \left.-2 \frac{\lambda^{2}}{\alpha^{3}} \xi^{2}\left(t_{i} ; \beta\right) \xi^{\prime}\left(t_{i} ; \beta\right) \mathcal{C}_{3 i}\right] \\
& v_{24}=v_{42}=\sum_{i=1}^{n}\left[\frac{\lambda^{2}}{2 \alpha^{2}} \xi\left(t_{i} ; \beta\right) \xi^{\prime}\left(t_{i} ; \beta\right) \mathcal{C}_{1 i}-\frac{\lambda}{2 \alpha} \xi^{\prime}\left(t_{i} ; \beta\right) \mathcal{C}_{3 i}\right. \\
& \left.-\frac{\lambda^{2}}{2 \alpha^{2}} \xi\left(t_{i} ; \beta\right) \xi^{\prime}\left(t_{i} ; \beta\right) \mathcal{C}_{5 i}+\frac{\lambda}{2 \alpha} \xi^{\prime}\left(t_{i} ; \beta\right) \mathcal{C}_{6 i}\right] \text {, } \\
& v_{33}=\sum_{i=1}^{n}\left[\frac{\lambda^{2}}{\alpha^{2}} \xi^{4}\left(t_{i} ; \beta\right) \mathcal{C}_{1 i}+\frac{\lambda}{\alpha^{2}} \xi^{2}\left(t_{i} ; \beta\right) \mathcal{C}_{2 i}-2 \frac{\lambda}{\alpha^{3}} \xi^{3}\left(t_{i} ; \beta\right) \mathcal{C}_{3 i}\right], \\
& v_{34}=v_{43}=\sum_{i=1}^{n}\left[\frac{\lambda}{\alpha^{2}} \xi^{2}\left(t_{i} ; \beta\right)\left\{\mathcal{C}_{1 i}-\mathcal{C}_{2 i}\right\}+\frac{1}{2 \alpha} \xi^{2}\left(t_{i} ; \beta\right)\left\{\mathcal{C}_{6 i}-\mathcal{C}_{3 i}\right\}\right] \text {, } \\
& v_{44}=\frac{1}{4} \sum_{i=1}^{n}\left[\mathcal{C}_{1 i}+\mathcal{C}_{4 i}-2 \mathcal{C}_{5 i}\right]
\end{aligned}
$$

in which

$$
\mathcal{C}_{1 i}=\operatorname{Var}\left(\tau_{i} \mid \mathbf{t}\right), \quad \mathcal{C}_{2 i}=\operatorname{Var}\left(\tau_{i} \gamma_{i} \mid \mathbf{t}\right), \quad \mathcal{C}_{3 i}=\operatorname{Cov}\left(\tau_{i}, \tau_{i} \gamma_{i} \mid \mathbf{t}\right)
$$


$\mathcal{C}_{4 i}=\operatorname{Var}\left(\log \tau_{i} \mid \mathbf{t}\right), \quad \mathcal{C}_{5 i}=\operatorname{Cov}\left(\tau_{i}, \log \tau_{i} \mid \mathbf{t}\right), \quad \mathcal{C}_{6 i}=\operatorname{Cov}\left(\tau_{i} \gamma_{i}, \log \tau_{i} \mid \mathbf{t}\right)$,

whose values are given in Appendix C.

Applying (23) and (24), we obtain the observed information as

$$
I^{*}(\hat{\boldsymbol{\theta}} ; \mathbf{t})=\ell_{c}^{*}(\hat{\boldsymbol{\theta}} ; \boldsymbol{t})-\ell_{m}^{*}(\hat{\boldsymbol{\theta}} ; \boldsymbol{t}) .
$$

The standard errors of the MLEs of the EM-algorithm are the square root of the diagonal elements of the $I^{*-1}(\hat{\boldsymbol{\theta}} ; \mathbf{t})$.

\section{An Illustrative Example}

To illustrate the model proposed in this paper, we use the daily ozone concentrations data used by Vilca et al. (2011) (Nadarajah, 2008), provided by the New York State Department of Conservation. We assume that the data are uncorrelated and, therefore, a diurnal or cyclic trend analysis is not necessary. This assumption has been supported by several authors for different reasons, for example, environmental data are sometimes reported as averages and so there is no spatial-time dependence (Gokhale and Khare, 2007, and Vilca et al., 2011). A descriptive summary of this data set including the mean, median, standard deviation (SD), CV, CS and CK is given in Table 1.

Table 1. Descriptive statistics for the ozone data.

\begin{tabular}{cccccccccc}
\hline \hline Median & \multirow{2}{*}{ Mean } & SD & CV & CS & CK & Range & Min. & Max. & $\mathrm{n}$ \\
\hline 31.50 & \multirow{2}{*}{42.13} & 32.99 & 78.30 & 1.21 & 3.11 & 167.00 & 1.00 & 168.00 & 116 \\
\hline
\end{tabular}

An exploratory data analysis based on this table indicate a positively skewed distribution with a kurtosis greater than three. Vilca et al. (2011) show that the $S N-B S$ distribution gives a better fit than the ordinary $B S$ distribution. We fit the $S N T-B S$ distribution to this data set and obtain the ML estimates (MLEs) of the parameters. To compare the fitted results, we compute the maximized log-likelihood $(\hat{\ell})$, the Akaike information criterion (AIC) and the Bayesian information criterion (BIC). Results along with standard errors (SE) of the MLEs are displayed in Table 2. 

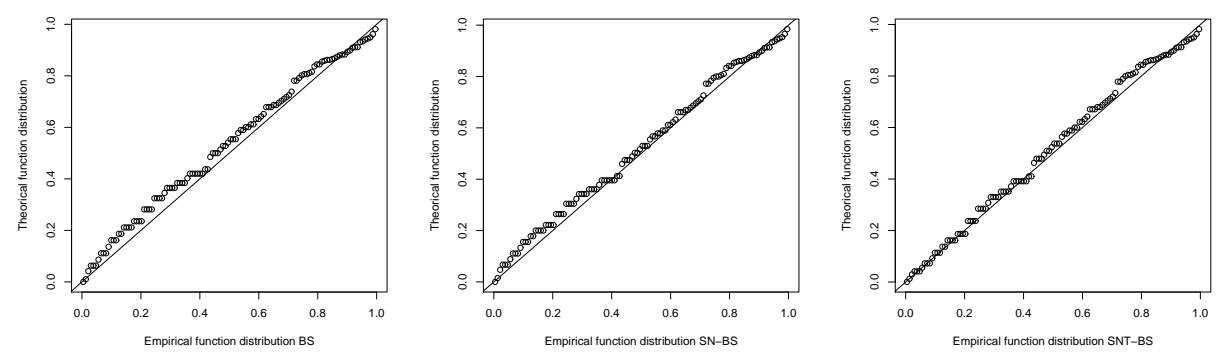

Figure 2. The pp-plot for the $B S, S N-B S$ and $S N T-B S$ models.

Table 2. Fitted various models on the ozone data set.

\begin{tabular}{|c|c|c|c|c|c|c|}
\hline \multirow[b]{2}{*}{ parameter } & \multicolumn{2}{|c|}{$B S$} & \multicolumn{2}{|c|}{$S N-B S$} & \multicolumn{2}{|c|}{$S N T-B S$} \\
\hline & MLE & $\mathrm{SE}$ & MLE & $\mathrm{SE}$ & MLE & $\mathrm{SE}$ \\
\hline$\alpha$ & 0.98201 & 0.06447 & 1.27025 & 0.23613 & 1.52074 & 0.21616 \\
\hline$\beta$ & 28.0231 & 2.26357 & 14.8352 & 4.02982 & 11.3178 & 2.10716 \\
\hline$\lambda$ & - & - & 1.06675 & 0.53475 & 4.37207 & 2.78484 \\
\hline$\nu$ & - & - & - & - & 1.54281 & 0.94345 \\
\hline$\ell(\hat{\boldsymbol{\theta}})$ & \multicolumn{2}{|c|}{-549.0971} & \multicolumn{2}{|c|}{-545.6057} & \multicolumn{2}{|c|}{-541.1762} \\
\hline AIC & \multicolumn{2}{|c|}{1102.194} & \multicolumn{2}{|c|}{1097.211} & \multicolumn{2}{|c|}{1090.352} \\
\hline $\mathrm{BIC}$ & \multicolumn{2}{|c|}{1107.701} & \multicolumn{2}{|c|}{1105.472} & \multicolumn{2}{|c|}{1101.367} \\
\hline
\end{tabular}

According to the AIC and the BIC, we see that the $S N T-B S$ distribution is the best among the fitted models. The PP-plots are presented in Figure 2 .

To study the validity of the three hypothetical models, we obtain the Kolmogorov-Smirnov (K-S) goodness of fit test. The procedure for calculating the K-S test statistic $D_{n}$, which is defined as the maximum value of the absolute difference between the empirical and estimated cumulative distributions, and the corresponding $p$-values are described below:

Step 1: Order the $n$ data values and called them $t_{(1)}, t_{(2)}, \ldots, t_{(n)}$.

Step 2: Compute the K-S test statistic

$$
D_{n}=\max _{j=1, \ldots, n}\left\{\frac{j}{n}-\hat{F}\left(t_{(j)}\right), \hat{F}\left(t_{(j)}\right)-\frac{j-1}{n}\right\},
$$

where $\hat{F}(\cdot)$ is the fitted cdf of a hypothetical distribution. 
Step 3: For $i=1,2, \ldots, N$, generate $n$ random numbers from $U(0,1)$ and order them to obtain $u_{(1)}^{(i)} \leqslant u_{(2)}^{(i)} \leqslant \ldots \leqslant u_{(n)}^{(i)}$.

Step 4: Compute

$$
d^{(i)}=\max _{j=1, \ldots, n}\left\{\frac{j}{n}-u_{(j)}^{(i)}, u_{(j)}^{(i)}-\frac{j-1}{n}\right\}
$$

Step 5: Let $I_{i}=1$ if $d^{(i)} \geqslant D_{n}$ and 0 otherwise. The $p$-value is estimated by $\frac{1}{N} \sum_{i=1}^{N} I_{i}$.

Results are listed in Table 3.

Table 3. The K-S test results for the fitted models.

\begin{tabular}{lccc}
\hline \hline & \multicolumn{3}{c}{ Model } \\
\cline { 2 - 4 } & $B S$ & $S N-B S$ & $S N T-B S$ \\
\hline$D_{n}$ & 0.398411 & 0.066425 & 0.063735 \\
$p$-value & 0.000001 & 0.650123 & 0.712314 \\
\hline
\end{tabular}

The reported $p$-values can be used as a similarity assessment of the experimental data against the fitted distribution. Of the three models, the best fit is $S N T-B S$ with a $p$-value of 0.712314 . This strongly suggests that the ozone data follow a $S N T-B S$ distribution.

In Figure 3, we plot the profile log-likelihood of the degrees of freedom $\nu$ for the $S N T-B S$ model. It is obvious that the $S N-B S$ model is not suitable for this data set since the profile log-likelihood has a significant drop at the peak value of $\nu=1.54281$.

\section{Simulation Study}

First, we conduct a simulation study to compare the fitting performance of the existing ordinary $B S$ and $S N-B S$ models with the proposed $S N T-B S$ model. Vilca et al. (2011) have developed an efficient ECM algorithm for the ML fitting of $S N-B S$ distribution. They showed that, the $S N-B S$ distribution has a convenient hierarchical representation:

$$
T \mid(H=h) \sim E B S\left(\alpha_{\delta}, \beta, \sigma=2, \lambda_{h}\right), \quad H \sim T N(0,1 ;(0, \infty)),
$$




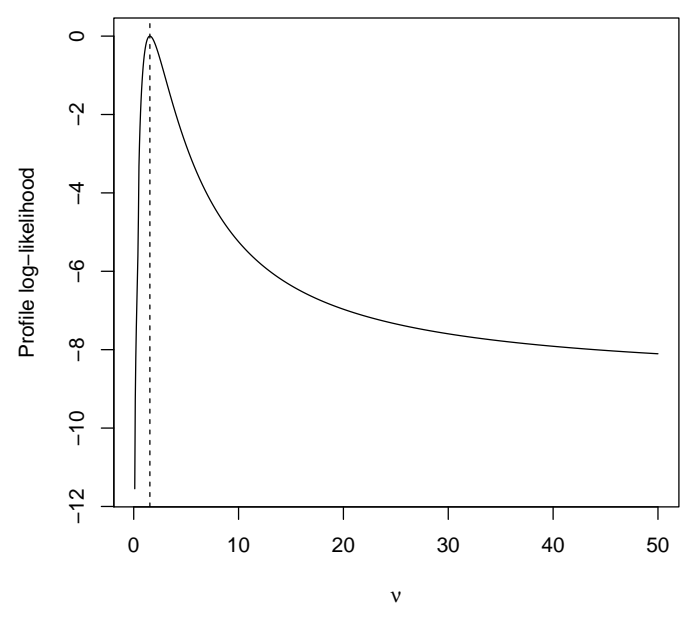

Figure 3. Plot of the profile $\log$-likelihood for $\nu$.

where $\alpha_{\delta}=\alpha \sqrt{1-\delta^{2}}, \lambda_{h}=-\frac{\delta h}{\sqrt{1-\delta^{2}}}$ and $\delta=\frac{\lambda}{\sqrt{1+\lambda^{2}}}$. We note that if $\lambda=0$, then both $S N T-B S$ and $S N-B S$ models reduce to the $B S$ model.

As in Ho et al. (2011), for $n=250,500,1000,5000$ we generated synthetic data from the BS distribution, when the parameters were set to $\alpha=1$ and $\beta=1$ to produce highly skewed distributions.

Then, to create heavy tails, we increased the size of each sample by $2 \%$ adding data generated by a uniform distribution over the interval $[10,20]$ to each simulated data set. So the simulation sample sizes become 255, 510, 1020 and 5100. Simulations were run with a total of 500 replications for each sample size.

The $B S, S N-B S$ and $S N T-B S$ distributions were fitted to each simulated data set using the ECM algorithm. The initial values for the parameters of the various models in each sample were obtained by the procedure $D E$ optim in the statistical software $R$. In each trail, we recorded log-likelihoods $(\ell(\hat{\boldsymbol{\theta}}))$, AIC and BIC values. All computations were carried out using the statistical software $R$ version 3.0.2. Table 4 presents the average log-likelihood, AIC and BIC values the amount of iterations for various sample sizes. We see that the $S N T-B S$ model has significantly better performance when the sample size becomes large $(n>500)$. 
Table 4. Comparison of average log-likelihood, AIC and BIC for $B S, S N-B S$ and $S N T-B S$ models under various sample sizes.

\begin{tabular}{ccccc}
\hline \hline & & \multicolumn{3}{c}{ Model } \\
\cline { 3 - 5 } $\mathrm{n}$ & Criterion & $B S$ & $S N-B S$ & $S N T-B S$ \\
\hline \multirow{2}{*}{255} & $-\ell(\hat{\boldsymbol{\theta}})$ & 370.2174 & 366.5192 & 363.5563 \\
& $\mathrm{AIC}$ & 744.4349 & 739.0385 & 735.1126 \\
& $\mathrm{BIC}$ & 751.5174 & 749.6623 & 749.2777 \\
510 & $-\ell(\hat{\boldsymbol{\theta}})$ & 745.5182 & 738.6540 & 733.8512 \\
& $\mathrm{AIC}$ & 1495.036 & 1483.308 & 1475.702 \\
& $\mathrm{BIC}$ & 1503.505 & 1496.011 & 1492.640 \\
\multirow{2}{*}{1020} & $-\ell(\hat{\boldsymbol{\theta}})$ & 1489.656 & 1476.135 & 1468.165 \\
& $\mathrm{AIC}$ & 2983.312 & 2958.270 & 2944.331 \\
& $\mathrm{BIC}$ & 2993.167 & 2973.053 & 2964.041 \\
& & & & \\
\multirow{2}{*}{5100} & $-\ell(\hat{\boldsymbol{\theta}})$ & 7476.361 & 7407.631 & 7370.001 \\
& $\mathrm{AIC}$ & 14956.72 & 14821.26 & 14748.01 \\
& $\mathrm{BIC}$ & 14969.80 & 14840.87 & 14774.15 \\
\hline
\end{tabular}

Next, we use Monte Carlo simulations to evaluate the finite-sample performance of the ML estimates of the $S N T-B S$ distribution parameters determined from the ECM algorithm described in Section 3. The sample sizes and true values of the parameters considered were $n=10,20,40,60$, $\alpha=0.25,0.5,0.75,1.0,1.25, \lambda=0.2,1,1.5$ and $\nu=2,3$, while the scale parameter was fixed without loss of generality at $\beta=1$. The number of Monte Carlo replications was taken once again as $M=3000$. In order to examine the performance of the ML estimates, we compute for each sample size and for each estimate, denoted by $\hat{\theta}_{j}$, the mean, denoted by $E\left[\hat{\theta}_{j}\right]$, the relative bias $(R B)$ in absolute value, defined as $R B_{j}=\left|\left\{E\left[\hat{\theta}_{j}\right]-\theta_{j}\right\} / \theta_{j}\right|$, and the root mean square error $(M S E)$, defined by $M S E_{j}=E\left[\left\{\hat{\theta}_{j}-\theta_{j}\right\}^{2}\right]$, for $j=1,2,3,4$ where $\theta_{1}=\alpha, \theta_{2}=\beta, \theta_{3}=\lambda$ and $\theta_{4}=\nu$. Here the $M S E$ is the mean square error computed from the 3000 Monte Carlo replications. Tables 5-10 present the mean, $R B$ and $M S E$ of the ML estimates of the parameters $\alpha, \beta, \lambda$ and $\nu$. It can be observed that the $R B$ and $M S E$ become smaller as the sample size $n$ increases, as one would expect. 


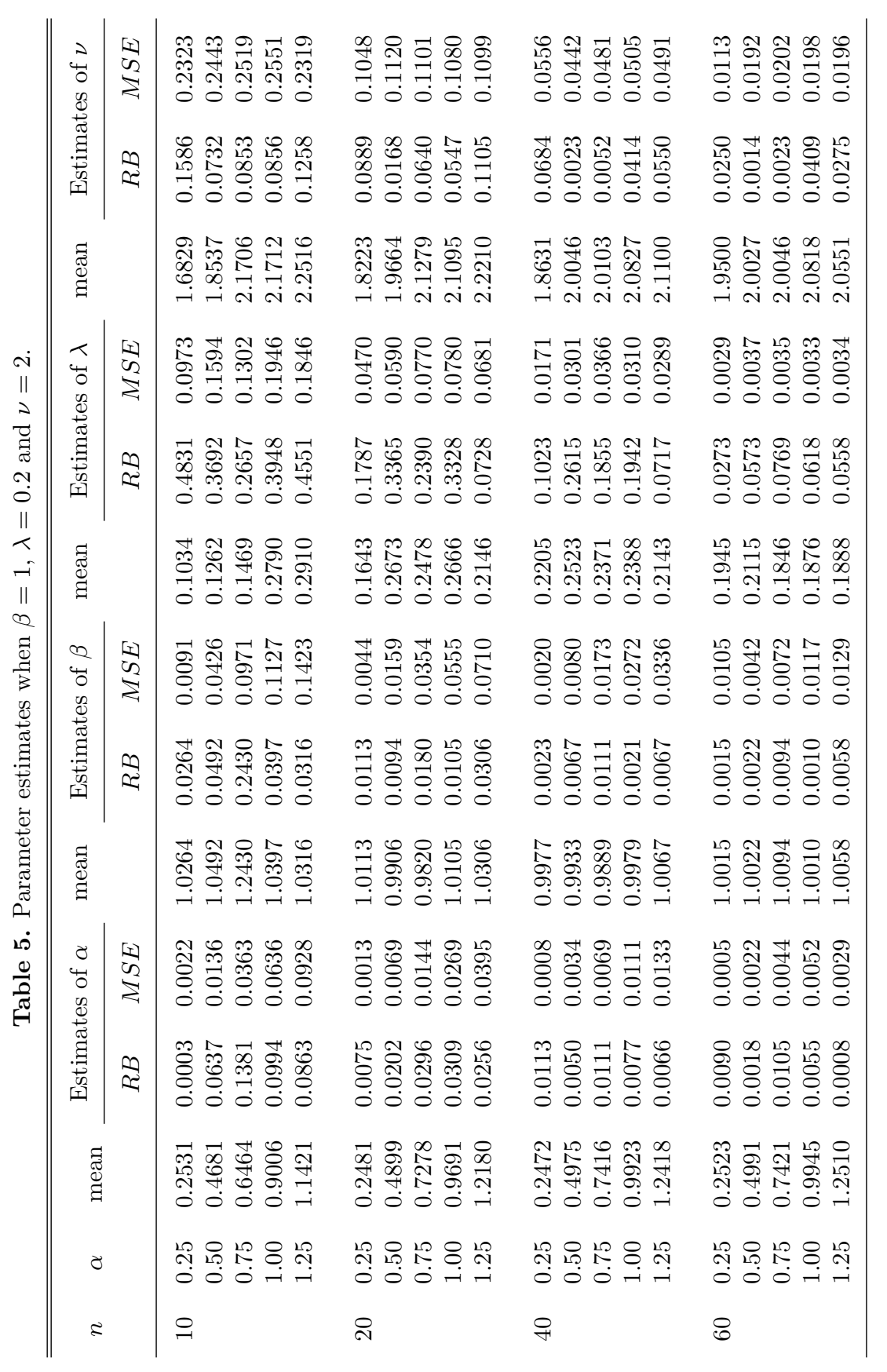




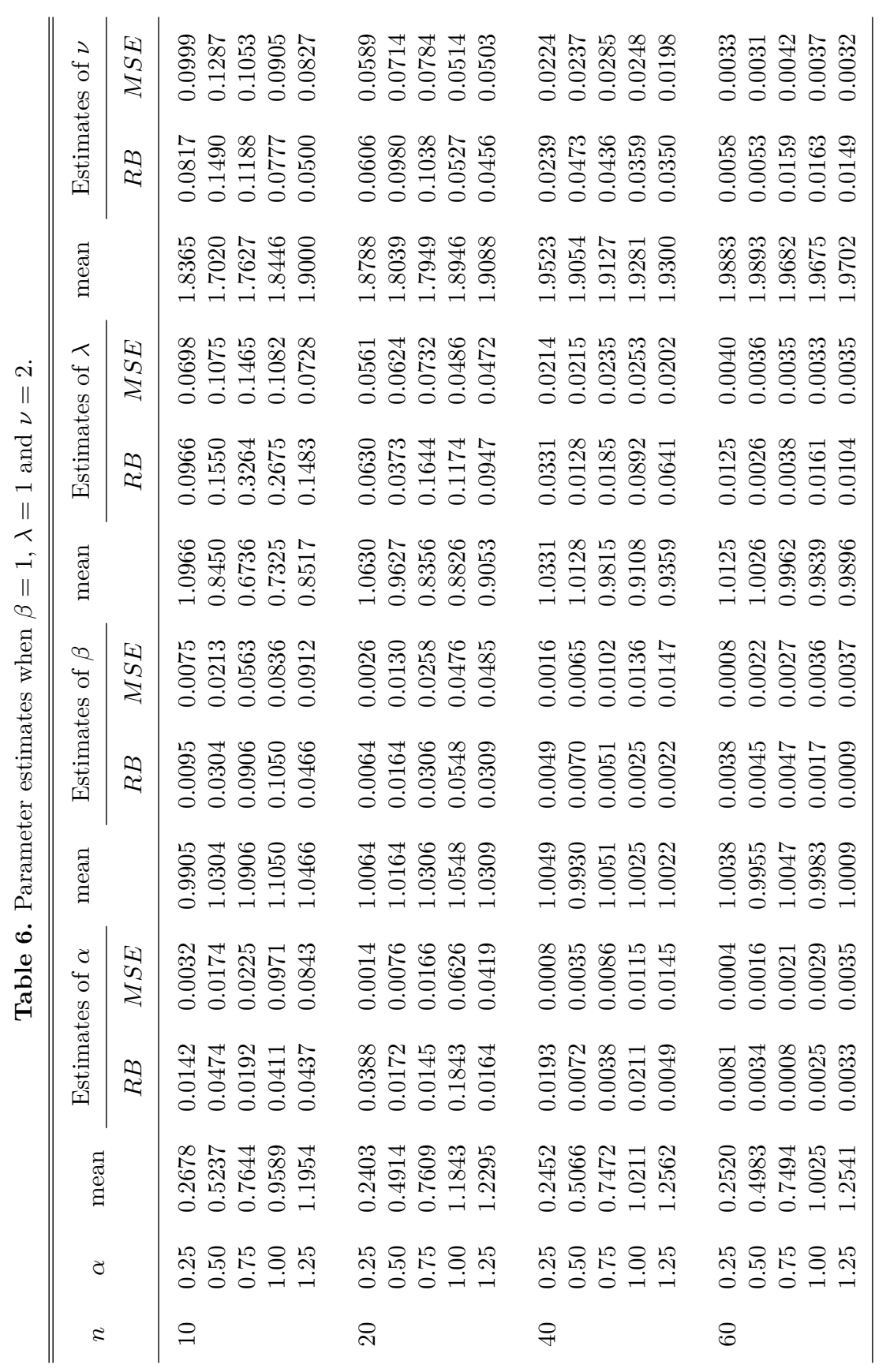




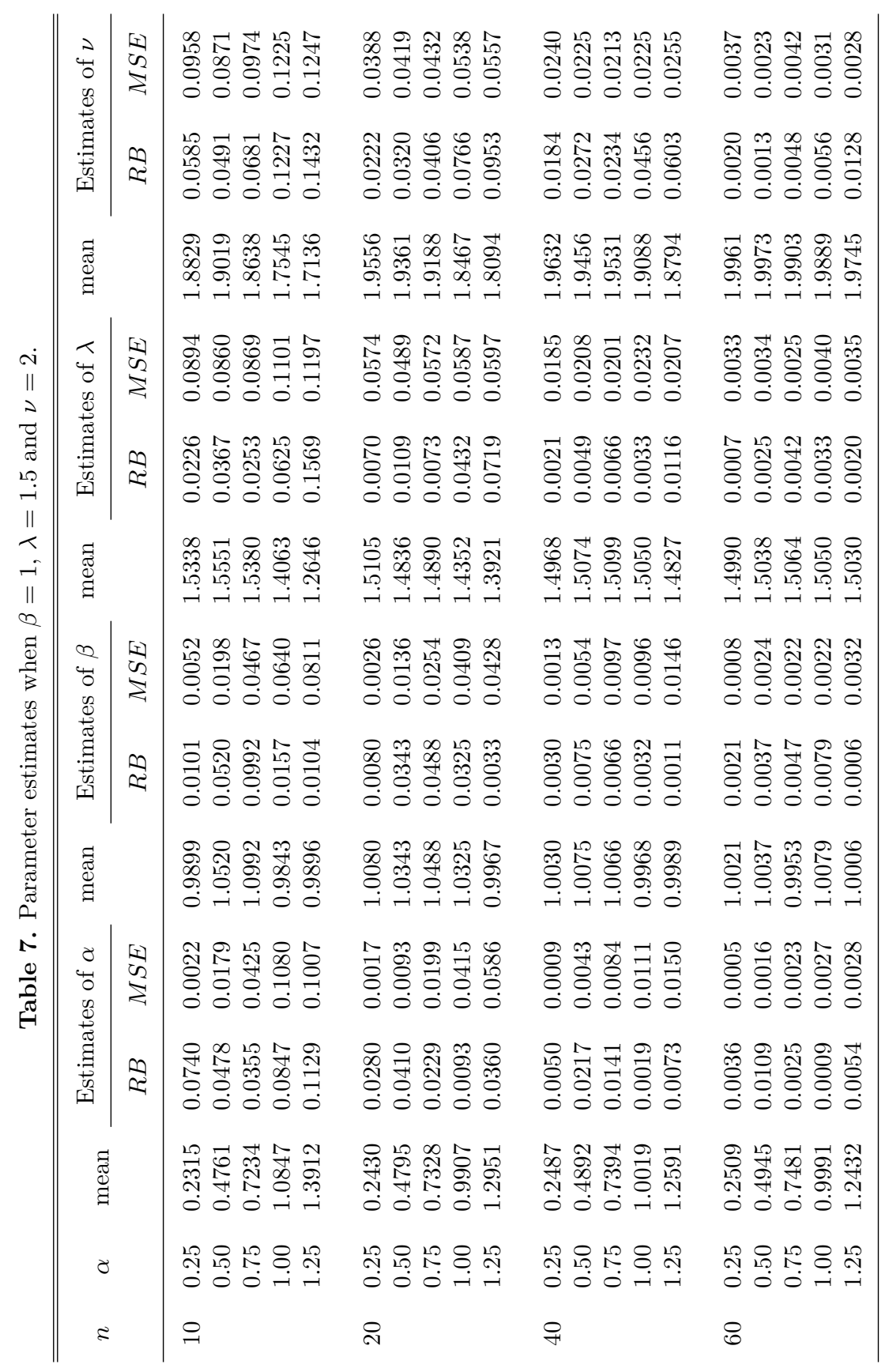




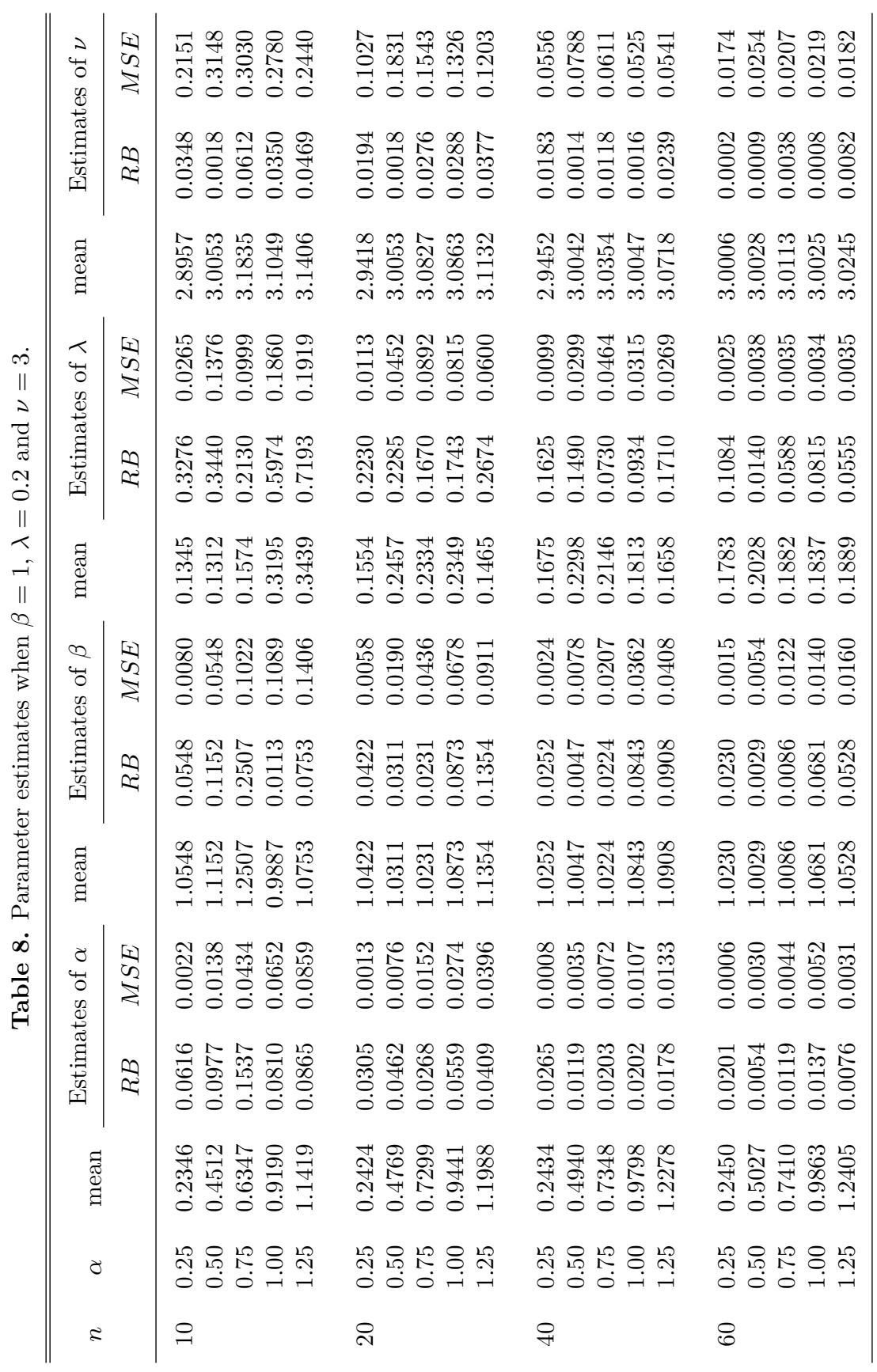




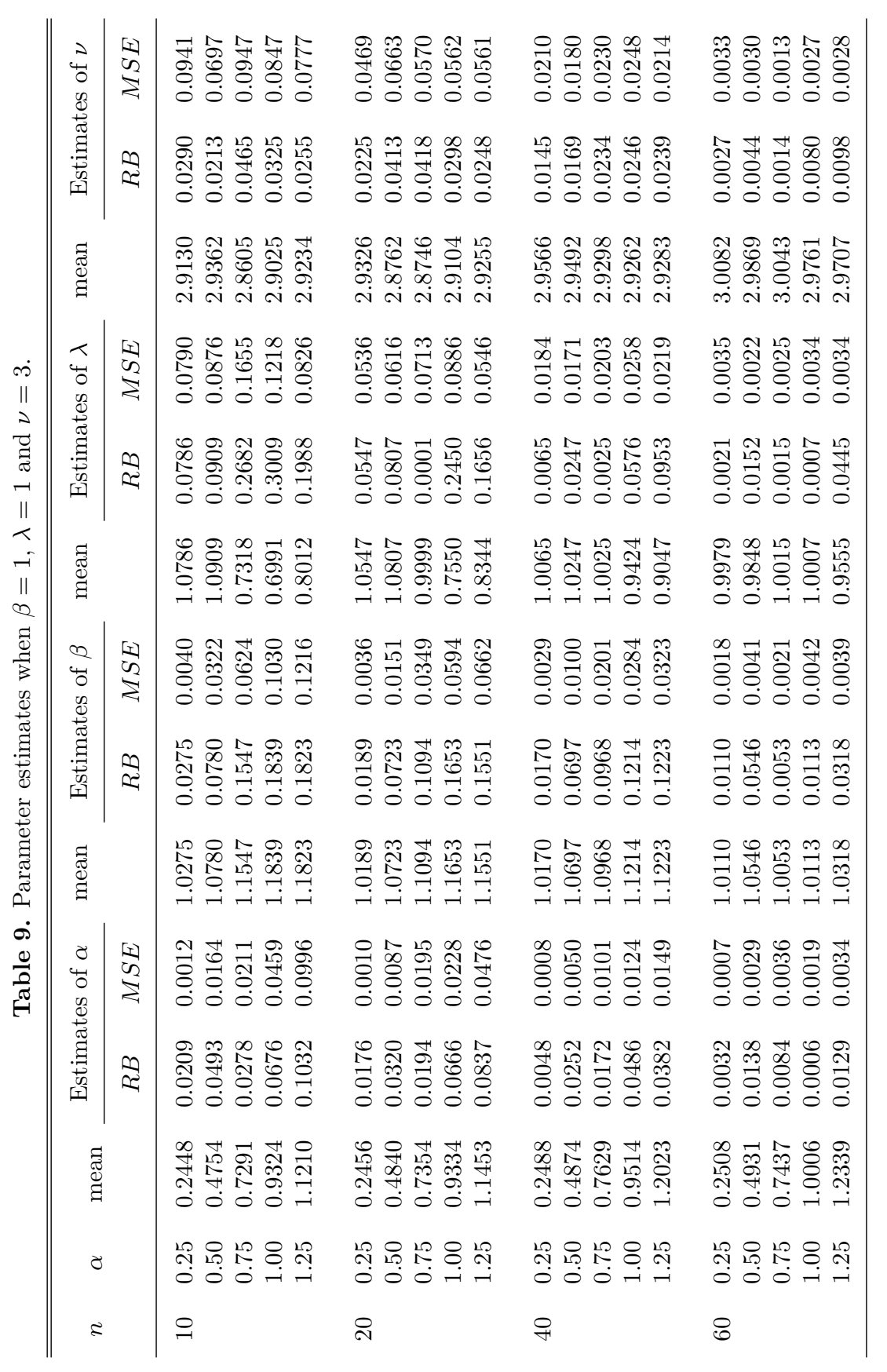




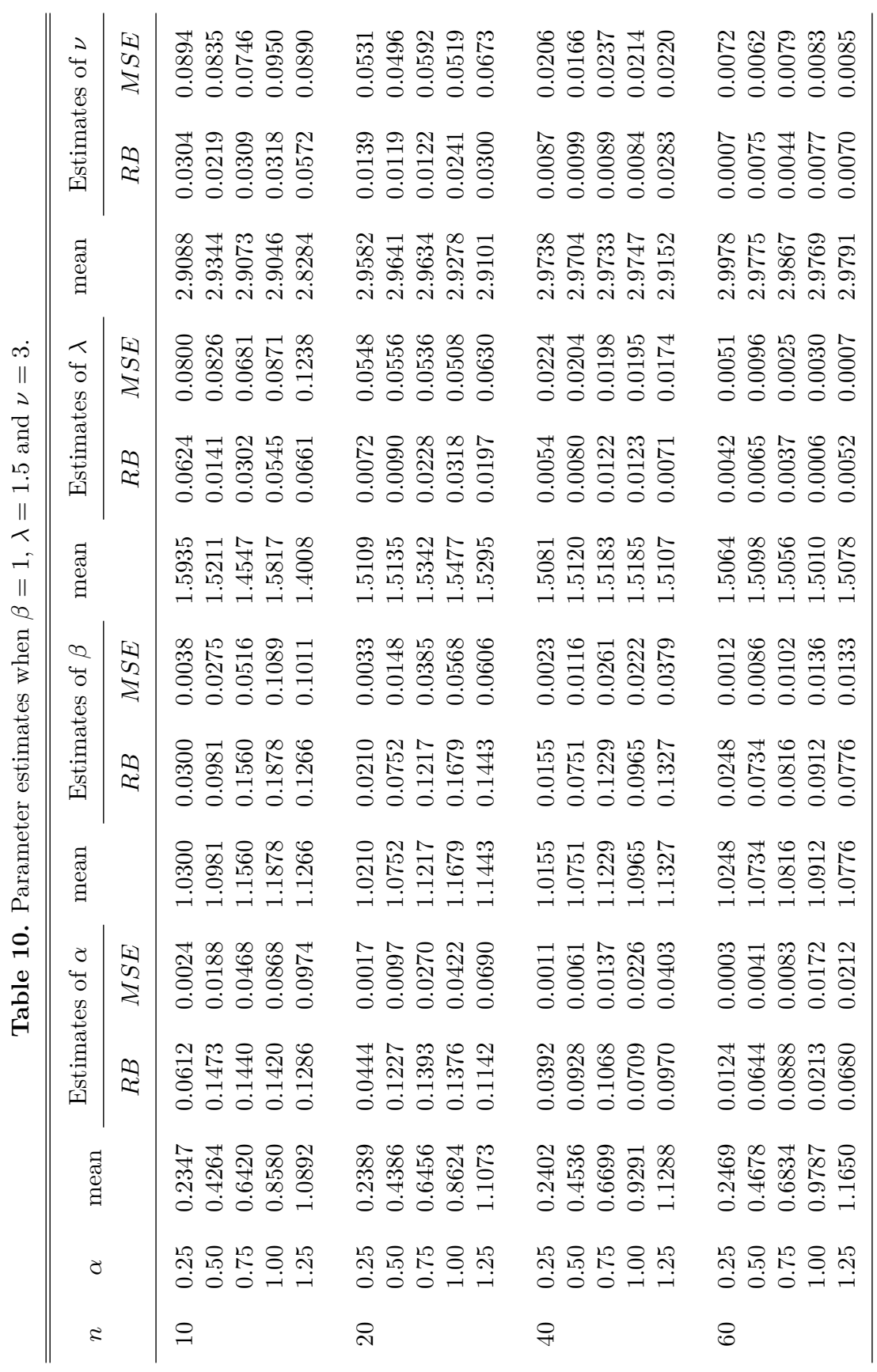




\section{Concluding Remarks}

We introduced an extension of the $B S$ model based on the $S N T$ distribution, called the $S N T-B S$ distribution and obtained several properties of this distribution such as moments, maximum likelihood estimation procedure via an EM-algorithm and a method to evaluate standard errors using the EMalgorithm. A real data set was used to show flexibility of the proposed model. Two simulation studies are used to illustrate the performance of the proposed distribution.

Several extensions of the $B S$ model can be considered, and we hope to study these in the near future.

\section{Acknowledgement}

The authors are grateful to the editor and two anonymous referees for their many helpful comments and suggestions on an earlier version of this paper which led to this improved version. Also, the authors are grateful to Sobhan Shafiei (PhD student in Shahid Bahonar University of Kerman) for computational assistance.

\section{References}

Azzalini, A. (1985). A Class of Distributions which Includes the Normal Ones. Scandinavian Journal of Statistis, 12, 171-178.

Azzalini, A. (1986). Further Results on a Class of Distributions which Includes the Normal Ones. Statistica, 46, 199-208.

Azzalini, A. and Capitanio, A. (2003). Distributions Generated by Perturbation of Symmetry with Emphasis on a Multivariate Skew- $t$ Distribution. Journal of the Royal Statistical Society: Series B , 65, 367-389.

Barros, M., Paula, G.A. and Leiva, V. (2008). A New Class of Survival Regression Models with Heavy-tailed Errors: Robustness and Diagnostics. Lifetime Data Analysis, 14, 316-332.

Birnbaum, Z.W. and Saunders, S.C. (1969). A New Family of Life Distributions. Journal of Applied Probability, 6, 319-327.

Cordeiro, G.M. and Lemonte, J.L. (2011). The $\beta$-Birnbaum-Saunders Distribution: An Improved Distribution for Fatigue Life Modeling. Computational Statistics and Data Analysis, 55, 1445-1461. 
Dempster, A.P., Laird, N.M. and Rubim, D.B. (1977). Maximum Likelihood from Incomplete Data Via the EM Algorithm (with discussion). Journal of the Royal Statistical Society: Series $B, \mathbf{3 9}, 1-38$.

Desmond, A. (1985). Stochastic Models of Failure in Random Environments. Canadian Journal of Statistics, 13, 171-183.

Díaz-García, J.A. and Leiva-Sánchez, V. (2005). A New Family of Life Distributions Based on the Elliptically Contoured Distributions. Journal of Statistical Planning and Inference, 128, 445-457.

Gokhale, S. and Khare, M. (2007). Statistical Behavior of Carbo nmonoxide from Vehicular Exhausts in Urban Environments. Environmental Modelling and Software, 22, 526-535.

Gómez, H.W., Olivares, J. and Bolfarine, H. (2009). An Extension of the Generalized Birnbaum-Saunders Distribution. Statistics and Probability Letters, 79, 331-338.

Guiraud, P., Leiva, V. and Fierro, R. (2009). A Non-Central Version of the BirnbaumSaunders Distribution for Reliability Analysis. IEEE Transaction Reliability, 58, 152-160.

Henze, N.A. (1986). A Probabilistic Representation of the Skew-Normal Distribution. Scandinavian Journal of Statistics, 13, 271-275.

Ho, H.J., Pyne, S. and Lin, T.I. (2011). Maximum Likelihood Inference for Mixtures of Skew Student-t-Normal Distributions through Practical EM-type Algorithms. Statistics and Computing, 22, 287-299.

Leiva, V., Barros, M., Paula, G.A. and Galea, M. (2007). Influence Diagnostics in LogBirnbaum-Saunders Regression Models with Censored Data. Computational Statistics and Data Analysis, 51, 5694-5707.

Leiva, V., Hernández, H. and Riquelme, M. (2006). A New Package for the Birnbaum-Saunders Distribution. $R$ Journal, 6, 35-40. http://www.rproject.org/doc/Rnews/Rnews.pdf.

Leiva, V., Vilca, F., Balakrishnan, N. and Sanhueza, A. (2010). A Skewed Sinh-Normal Distribution and Its Properties and Application to Air Pollution. Communications in StatisticsTheory and Methods, 39, 426-443.

Lemonte, J.L. (2013). A New Extended Birnbaum-Saunders Regression Model for Lifetime Modeling. Computational Statistics and Data Analysis, 64, 34-50.

Louis, T.A. (1982). Finding the Observed Information Matrix when Using the EM Algorithm. Journal of the Royal Statistical Society B, 44, 226-233.

Meng, X.L. and Rubin, D.B. (1993). Maximum Likelihood Estimation Via the ECM Algorithm: A General Framework. Biometrika, 80, 267-278. 
Nadarajah, S. (2008). A Truncated Inverted Beta Distribution with Application to Air Pollution Data. Stochastic Environmental Research and Risk Assessment, 22, 285-289.

Nadarajah, S. and Kotz, S. (2003). Skewed Distributions Generated by the Normal Kernel. Statistics and Probability Letters, 65, 269-277.

Podlaski, R. (2008). Characterization of Diameter Data in Near-Natural Forests Using the Birnbaum-Saunders Distribution. Canadian Journal of Forest Research, 38, 518-527.

Sanhueza, A., Leiva, V. and Balakrishnan, N. (2008) The Generalized Birnbaum-Saunders Distribution and Its Theory, Methodology and Application. Communications in StatisticsTheory and Methods, 37, 645-670.

Vilca, F. and Leiva, V. (2006) A New Fatigue Life Model Based on the Family of Skew Elliptical Distributions. Communications in Statistics-Theory and Methods, 35, 229-244.

Vilca, F., Sanhueza, A., Leiva, V. and Christakos, G. (2010). An Extended BirnbaumSaunders Model and Its Application in the Study of Environmental Quality in Santiago, Chile. Stochastic Environmental Research and Risk Assessment, 24, 771-782.

Vilca, F., Santana, L., Leiva, V. and Balakrishnan, N. (2011). Estimation of Extreme Percentiles in Birnbaum-Saunders Distributions. Computational Statistics and Data Analysis, 55, 1665-1678.

\section{Appendix A}

To prove (6) we use the following stochastic representation for the SN model (see for example Henze, 1986)

$$
\frac{1}{\sqrt{1+\lambda^{2}}} Y_{1}+\frac{\lambda}{\sqrt{1+\lambda^{2}}}\left|Y_{0}\right| \sim S N(\lambda)
$$

Using this representation, it is clear that $Z \mid \tau \sim S N(\lambda \sqrt{\tau})$. By the law of total probability and using Proposition 1, the pdf of the r.v. $Z$ is readily obtained as

$$
f_{S N T}(z ; \lambda, \nu)=\int_{0}^{\infty} 2 \phi(z) \Phi(\lambda \sqrt{\tau} z) \gamma\left(\tau ; \frac{\nu}{2}, \frac{\nu}{2}\right) d \tau=2 \phi(z) T(\lambda z ; \nu),
$$

where $\gamma(\cdot ; \alpha, \beta)$ is the pdf of the gamma distribution with shape parameter $\alpha$ and scale parameter $\beta$. 


\section{Appendix B}

(a) Standard calculation yields

$$
\begin{aligned}
E(\tau \mid T=t)= & \int_{0}^{\infty} \tau f(\tau \mid t) d \tau \\
= & \int_{0}^{\infty} \frac{\left(\frac{\nu}{2}\right)^{\frac{\nu}{2}}}{\Gamma\left(\frac{\nu}{2}\right)} \tau^{\frac{\nu+2}{2}-1} \exp \left\{-\frac{\nu \tau}{2}\right\} \frac{\Phi(\lambda \sqrt{\tau} a(t ; \alpha, \beta))}{T(\lambda a(t ; \alpha, \beta) ; \nu)} \\
= & \frac{\Gamma\left(\frac{\nu+2}{2}\right)}{\frac{\nu}{2} \Gamma\left(\frac{\nu}{2}\right) T(\lambda a(t ; \alpha, \beta) ; \nu)} \\
& \times \int_{0}^{\infty} \gamma\left(\tau ; \frac{\nu+2}{2}, \frac{\nu}{2}\right) \Phi(\lambda \sqrt{\tau} a(t ; \alpha, \beta)) d \tau
\end{aligned}
$$

where $\gamma(\cdot ; \alpha, \beta)$ denotes the density of $\Gamma(\alpha, \beta)$. By Proposition 1, it suffices to show that

$$
E(\tau \mid T=t)=\frac{T\left(\lambda a(t ; \alpha, \beta) \sqrt{\frac{2}{\nu}+1} ; \nu+2\right)}{T(\lambda a(t ; \alpha, \beta) ; \nu)} .
$$

(b) Similarly, standard calculation yields

$$
\begin{aligned}
E & \left(\frac{\phi(\sqrt{\tau} \lambda a(t ; \alpha, \beta))}{\Phi(\sqrt{\tau} \lambda a(t ; \alpha, \beta))} \sqrt{\tau} \mid t\right) \\
= & \int_{0}^{\infty} \frac{\left(\frac{\nu}{2}\right)^{\frac{\nu}{2}}}{\Gamma\left(\frac{\nu}{2}\right)} \tau^{\frac{\nu+1}{2}-1} \exp \left\{-\frac{\nu \tau}{2}\right\} \frac{\phi(\lambda \sqrt{\tau} a(t ; \alpha, \beta))}{T(\lambda a(t ; \alpha, \beta) ; \nu)} d \tau \\
= & \frac{\left(\frac{\nu}{2}\right)^{\frac{\nu}{2}} \Gamma\left(\frac{\nu+1}{2}\right)}{\sqrt{2 \pi} \Gamma\left(\frac{\nu}{2}\right) T(\lambda a(t ; \alpha, \beta) ; \nu)} \\
& \times \int_{0}^{\infty} \frac{\tau^{\frac{\nu+1}{2}}-1}{\Gamma\left(\frac{\nu+1}{2}\right)} \exp \left\{-\frac{\left(\lambda^{2} a^{2}(t ; \alpha, \beta)+\nu\right)}{2} \tau\right\} d \tau \\
= & \frac{\left(\frac{\nu}{2}\right)^{\frac{\nu}{2}} \Gamma\left(\frac{\nu+1}{2}\right)}{\sqrt{2 \pi} \Gamma\left(\frac{\nu}{2}\right) T(\lambda a(t ; \alpha, \beta) ; \nu)\left(\frac{\lambda^{2} a^{2}(t ; \alpha, \beta)+\nu}{2}\right)^{\frac{\nu+1}{2}}} .
\end{aligned}
$$

(c) From (14), the expectation of a truncated normal distribution is given by

$$
E(\gamma \mid \tau, t)=\lambda a(t ; \alpha, \beta)+\frac{1}{\sqrt{\tau}} \frac{\phi(\sqrt{\tau} \lambda a(t ; \alpha, \beta))}{\Phi(\sqrt{\tau} \lambda a(t ; \alpha, \beta))} .
$$


Applying the law of iterated expectation and using Part (b) and (25), we get

$$
\begin{aligned}
E(\gamma \tau \mid t) & =E(\tau E(\gamma \mid \tau, t) \mid t) \\
& =\lambda a(t ; \alpha, \beta) E(\tau \mid t)+E\left(\frac{\phi(\sqrt{\tau} \lambda a(t ; \alpha, \beta))}{\Phi(\sqrt{\tau} \lambda a(t ; \alpha, \beta))} \sqrt{\tau} \mid t\right) .
\end{aligned}
$$

(d) Similarly, it is easy to verify that

$$
E\left(\gamma^{2} \mid \tau, t\right)=\lambda^{2} a^{2}(t ; \alpha, \beta)+\frac{1}{\tau}+\frac{\lambda a(t ; \alpha, \beta)}{\sqrt{\tau}} \cdot \frac{\phi(\sqrt{\tau} \lambda a(t ; \alpha, \beta))}{\Phi(\sqrt{\tau} \lambda a(t ; \alpha, \beta))} .
$$

Using Part (b) and (26), together with the law of iterated expectation gives

$$
\begin{aligned}
& E\left(\gamma^{2} \tau \mid t\right)=E(\left.\tau E\left(\gamma^{2} \mid \tau, t\right) \mid t\right) \\
&=\lambda^{2} a^{2}(t ; \alpha, \beta) E(\tau \mid t)+1 \\
& \quad+\lambda a(t ; \alpha, \beta) E\left(\frac{\phi(\sqrt{\tau} \lambda a(t ; \alpha, \beta))}{\Phi(\sqrt{\tau} \lambda a(t ; \alpha, \beta))} \sqrt{\tau} \mid t\right) .
\end{aligned}
$$

(e) From (16), we have

$$
\begin{aligned}
\frac{d}{d \tau} \int_{0}^{\infty} f(\tau \mid t) d \tau= & \frac{d}{d \tau} \int_{0}^{\infty} \frac{\left(\frac{\nu}{2}\right)^{\frac{\nu}{2}}}{\Gamma\left(\frac{\nu}{2}\right)} \tau^{\nu / 2-1} \\
& \times \exp \left\{-\frac{\nu \tau}{2}\right\} \frac{\Phi(\lambda \sqrt{\tau} a(t ; \alpha, \beta))}{T(\lambda a(t ; \alpha, \beta) ; \nu)}=0
\end{aligned}
$$

By Leibnitz's rule, we get

$$
\begin{aligned}
\log \left(\frac{\nu}{2}\right) & +\frac{\nu+1}{\nu}-D G\left(\frac{\nu+1}{2}\right)-\frac{1}{T(\lambda a(t ; \alpha, \beta) ; \nu)} \\
& \times \int_{-\infty}^{\lambda a(t ; \alpha, \beta)} g_{\nu}(x) t(x ; \nu) d x+E(\log \tau \mid t)-E(\tau \mid t)=0 .
\end{aligned}
$$

Hence

$$
\begin{aligned}
E(\log (\tau) \mid T=t)= & D G\left(\frac{\nu+1}{2}\right)-\log \frac{\nu}{2}-1+E(\tau \mid T=t)-\frac{1}{\nu} \\
& +\frac{1}{T(\lambda a(t ; \alpha, \beta) ; \nu)} \int_{-\infty}^{\lambda a(t ; \alpha, \beta)} g_{\nu}(x) t(x ; \nu) d x .
\end{aligned}
$$




\section{Appendix C}

All proofs of this Appendix are similar to those of Appendix B. We first show the following:

$$
\begin{aligned}
E\left(\tau^{2} \mid t\right)= & \int_{0}^{\infty} \tau^{2} f(\tau \mid t) d \tau \\
= & \int_{0}^{\infty} \frac{\left(\frac{\nu}{2}\right)^{\frac{\nu}{2}}}{\Gamma\left(\frac{\nu}{2}\right)} \tau^{\frac{\nu+4}{2}-1} \exp \left\{-\frac{\nu \tau}{2}\right\} \frac{\Phi(\lambda \sqrt{\tau} a(t ; \alpha, \beta))}{T(\lambda a(t ; \alpha, \beta) ; \nu)} \\
= & \frac{\Gamma\left(\frac{\nu+4}{2}\right)}{\left(\frac{\nu}{2}\right)^{2} \Gamma\left(\frac{\nu}{2}\right) T(\lambda a(t ; \alpha, \beta) ; \nu)} \\
& \times \int_{0}^{\infty} \gamma\left(\tau ; \frac{\nu+4}{2}, \frac{\nu}{2}\right) \Phi(\lambda \sqrt{\tau} a(t ; \alpha, \beta)) d \tau \\
= & \frac{\Gamma\left(\frac{\nu+4}{2}\right) T\left(\lambda a(t ; \alpha, \beta) \sqrt{\frac{4}{\nu}+1} ; \nu+4\right)}{\left(\frac{\nu}{2}\right)^{2} \Gamma\left(\frac{\nu}{2}\right) T(\lambda a(t ; \alpha, \beta) ; \nu)}
\end{aligned}
$$

where $\gamma(\cdot ; \alpha, \beta)$ denote the density of $\Gamma(\alpha, \beta)$. Therefore

$$
\operatorname{Var}(\tau \mid t)=E\left(\tau^{2} \mid t\right)-E^{2}(\tau \mid t) .
$$

By (26),

$$
\begin{aligned}
E\left(\gamma^{2} \tau^{2} \mid t\right)= & E\left(\tau^{2} E\left(\gamma^{2} \mid \tau, t\right) \mid t\right) \\
= & \lambda^{2} a^{2}(t ; \alpha, \beta) E\left(\tau^{2} \mid t\right)+E(\tau \mid t) \\
& \quad+\lambda a(t ; \alpha, \beta) E\left(\frac{\phi(\sqrt{\tau} \lambda a(t ; \alpha, \beta))}{\Phi(\sqrt{\tau} \lambda a(t ; \alpha, \beta))} \sqrt{\tau^{3}} \mid t\right),
\end{aligned}
$$

where

$$
\begin{aligned}
E & \left(\frac{\phi(\sqrt{\tau} \lambda a(t ; \alpha, \beta))}{\Phi(\sqrt{\tau} \lambda a(t ; \alpha, \beta))} \sqrt{\tau^{3}} \mid t\right) \\
= & \int_{0}^{\infty} \frac{\left(\frac{\nu}{2}\right)^{\frac{\nu}{2}}}{\Gamma\left(\frac{\nu}{2}\right)} \tau^{\frac{\nu+3}{2}-1} \exp \left\{-\frac{\nu \tau}{2}\right\} \frac{\phi(\lambda \sqrt{\tau} a(t ; \alpha, \beta))}{T(\lambda a(t ; \alpha, \beta) ; \nu)} d \tau \\
= & \frac{\left(\frac{\nu}{2}\right)^{\frac{\nu}{2}} \Gamma\left(\frac{\nu+3}{2}\right)}{\sqrt{2 \pi} \Gamma\left(\frac{\nu}{2}\right) T(\lambda a(t ; \alpha, \beta) ; \nu)} \int_{0}^{\infty} \frac{\tau^{\frac{\nu+3}{2}-1}}{\Gamma\left(\frac{\nu+3}{2}\right)} \exp \left\{-\frac{\left(\lambda^{2} a^{2}(t ; \alpha, \beta)+\nu\right)}{2} \tau\right\} d \tau \\
= & \frac{\left(\frac{\nu}{2}\right)^{\frac{\nu}{2}} \Gamma\left(\frac{\nu+3}{2}\right)}{\sqrt{2 \pi} \Gamma\left(\frac{\nu}{2}\right) T(\lambda a(t ; \alpha, \beta) ; \nu)\left(\frac{\lambda^{2} a^{2}(t ; \alpha, \beta)+\nu}{2}\right)^{\frac{\nu+3}{2}}} .
\end{aligned}
$$


So

$$
\operatorname{Var}(\tau \gamma \mid t)=E\left(\tau^{2} \gamma^{2} \mid t\right)-E^{2}(\tau \gamma \mid t)
$$

Also, we have

$$
\begin{aligned}
\operatorname{Cov}(\tau, \tau \gamma \mid t)= & E\left(\tau^{2} \gamma \mid t\right)-E(\tau \mid t) E(\tau \gamma \mid t) \\
= & E\left(\tau^{2} E(\gamma \mid \tau, t) \mid t\right)-E(\tau \mid t) E(\tau \gamma \mid t) \\
= & \lambda a(t ; \alpha, \beta) E\left(\tau^{2} \mid t\right)+E\left(\frac{\phi(\sqrt{\tau} \lambda a(t ; \alpha, \beta))}{\Phi(\sqrt{\tau} \lambda a(t ; \alpha, \beta))} \sqrt{\tau^{3}} \mid t\right) \\
& -E(\tau \mid t) E(\tau \gamma \mid t),
\end{aligned}
$$

where all conditional expectations have been obtained earlier.

To compute $\operatorname{Cov}(\tau, \log \tau \mid t)$ and $\operatorname{Cov}(\tau \gamma, \log \tau \mid t)$, we first note that,

$$
\begin{aligned}
E(\tau \log \tau \mid t)= & \int_{0}^{\infty} \tau \log \tau f(\tau \mid t) d \tau \\
= & \int_{0}^{\infty} \log \tau \frac{\left(\frac{\nu}{2}\right)^{\frac{\nu}{2}}}{\Gamma\left(\frac{\nu}{2}\right)} \tau^{\frac{\nu+2}{2}-1} \exp \left\{-\frac{\nu \tau}{2}\right\} \frac{\Phi(\lambda \sqrt{\tau} a(t ; \alpha, \beta))}{T(\lambda a(t ; \alpha, \beta) ; \nu)} d \tau \\
= & \frac{\Gamma\left(\frac{\nu+2}{2}\right) T\left(\lambda a(t ; \alpha, \beta) \sqrt{\frac{2}{\nu}+1} ; \nu+2\right)}{\frac{\nu}{2} \Gamma\left(\frac{\nu}{2}\right) T(\lambda a(t ; \alpha, \beta) ; \nu)} \\
& \times \int_{0}^{\infty} \log \tau \frac{\left(\frac{\nu}{2}\right)^{\frac{\nu+2}{2}}}{\Gamma\left(\frac{\nu+2}{2}\right)} \tau^{\frac{\nu+2}{2}-1} \\
& \quad \times \exp \left\{-\frac{\nu \tau}{2}\right\} \frac{\Phi(\lambda \sqrt{\tau} a(t ; \alpha, \beta))}{T\left(\lambda a(t ; \alpha, \beta) \sqrt{\frac{2}{\nu}+1} ; \nu+2\right)} d \tau .
\end{aligned}
$$

As in Appendix B item (e), it is easy to verify that 


$$
\begin{aligned}
E(\tau \log \tau \mid t)= & \frac{\Gamma\left(\frac{\nu+2}{2}\right) T\left(\lambda a(t ; \alpha, \beta) \sqrt{\frac{2}{\nu}+1} ; \nu+2\right)}{\frac{\nu}{2} \Gamma\left(\frac{\nu}{2}\right) T(\lambda a(t ; \alpha, \beta) ; \nu)} \\
& \times\left[D G\left(\frac{\nu+2}{2}\right)-\log \frac{\nu}{2}-\frac{\nu+2}{\nu}\right. \\
& +\frac{\Gamma\left(\frac{\nu+4}{2}\right) T\left(\lambda a(t ; \alpha, \beta) \sqrt{\frac{4}{\nu}+1} ; \nu+4\right)}{\frac{\nu}{2} \Gamma\left(\frac{\nu+2}{2}\right) T\left(\lambda a(t ; \alpha, \beta) \sqrt{\frac{2}{\nu}+1} ; \nu+2\right)} \\
& -\frac{M}{\nu^{2}+2 \nu} \frac{t(M ; \nu+2)}{T(M ; \nu+2)} \\
& \left.+\frac{1}{T(M ; \nu+2)} \int_{-\infty}^{M} g_{\nu}(x) t(x ; \nu+2) d x\right],
\end{aligned}
$$

where

$$
\begin{aligned}
g_{\nu}(x)= & D G\left(\frac{\nu+3}{2}\right)-D G\left(\frac{\nu+2}{2}\right)-1-\log \left(1+\frac{x^{2}}{\nu+2}\right) \\
& +\frac{(\nu+3) x^{2}}{(\nu+2)\left(\nu+2+x^{2}\right)}
\end{aligned}
$$

and $M=\sqrt{\frac{2}{\nu}+1} \lambda a(t ; \alpha, \beta)$.

Also, by using the conditional expectation rule we have

$$
\begin{aligned}
E(\gamma \tau \log \tau \mid t)= & E(\tau \log \tau E(\gamma \mid \tau, t) \mid t) \\
= & \lambda a(t ; \alpha, \beta) E(\tau \log \tau \mid t) \\
& +\lambda a(t ; \alpha, \beta) E\left(\frac{\phi(\sqrt{\tau} \lambda a(t ; \alpha, \beta))}{\Phi(\sqrt{\tau} \lambda a(t ; \alpha, \beta))} \sqrt{\tau} \log \tau \mid t\right)
\end{aligned}
$$

The first conditional expectation has just been computed. The second con- 
ditional expectation is

$$
\begin{aligned}
E & \left(\frac{\phi(\sqrt{\tau} \lambda a(t ; \alpha, \beta))}{\Phi(\sqrt{\tau} \lambda a(t ; \alpha, \beta))} \sqrt{\tau} \log \tau \mid t\right) \\
= & \int_{0}^{\infty} \frac{\left(\frac{\nu}{2}\right)^{\frac{\nu}{2}}}{\Gamma\left(\frac{\nu}{2}\right)} \tau^{\frac{\nu+1}{2}-1} \exp \left\{-\frac{\nu \tau}{2}\right\} \frac{\phi(\lambda \sqrt{\tau} a(t ; \alpha, \beta))}{T(\lambda a(t ; \alpha, \beta) ; \nu)} \log \tau d \tau \\
= & \frac{\left(\frac{\nu}{2}\right)^{\frac{\nu}{2}} \Gamma\left(\frac{\nu+1}{2}\right)}{\sqrt{2 \pi} \Gamma\left(\frac{\nu}{2}\right) T(\lambda a(t ; \alpha, \beta) ; \nu)\left(\frac{\lambda^{2} a^{2}(t ; \alpha, \beta)+\nu}{2}\right)^{\frac{\nu+1}{2}}} \\
& \times \int_{0}^{\infty} \log \tau \frac{\left(\frac{\lambda^{2} a^{2}(t ; \alpha, \beta)+\nu}{2}\right)^{\frac{\nu+1}{2}} \tau^{\frac{\nu+1}{2}-1}}{\Gamma\left(\frac{\nu+1}{2}\right)} \exp \left\{-\frac{\left(\lambda^{2} a^{2}(t ; \alpha, \beta)+\nu\right)}{2} \tau\right\} \\
= & \sqrt{2 \pi} \Gamma\left(\frac{\nu}{2}\right) T(\lambda a(t ; \alpha, \beta) ; \nu)\left(\frac{\lambda^{2} a^{2}(t ; \alpha, \beta)+\nu}{2}\right)^{\frac{\nu+1}{2}} \Gamma\left(\frac{\nu+1}{2}\right) \\
& \times\left[D G\left(\frac{\nu+1}{2}\right)-\log \frac{\lambda^{2} a^{2}(t ; \alpha, \beta)+\nu}{2}-\frac{\nu+1}{\lambda^{2} a^{2}(t ; \alpha, \beta)+\nu}\right. \\
& +\frac{\Gamma\left(\frac{\nu+3}{2}\right)}{\left.\Gamma\left(\frac{\nu+1}{2}\right) \frac{\lambda^{2} a^{2}(t ; \alpha, \beta)+\nu}{2}\right] .}
\end{aligned}
$$

Using Proposition 2, we get

$$
\operatorname{Cov}(\tau, \log \tau \mid t)=E(\tau \log \tau \mid t)-E(\tau \mid t) E(\log \tau \mid t) .
$$

and

$$
\operatorname{Cov}(\tau \gamma, \log \tau \mid t)=E(\tau \gamma \log \tau \mid t)-E(\tau \gamma \mid t) E(\log \tau \mid t)
$$

Also,

$$
\operatorname{Var}(\log \tau \mid t)=E(\log \tau \mid t)^{2}-E^{2}(\log \tau \mid t) .
$$

$E(\log \tau \mid t)^{2}$ must be computed by the numerical methods.

\section{Farzaneh Hashemi}

Department of Statistics,

Faculty of Mathematics and Computer, Shahid Bahonar University of Kerman, Kerman, Iran.

email: farzane.hashemi1367@yahoo.com

\section{Vahid Amirzadeh}

Department of Statistics,

Faculty of Mathematics and Computer, Shahid Bahonar University of Kerman, Kerman, Iran.

email:vham51@yahoo.com 


\section{Ahad Jamalizadeh}

Department of Statistics,

Faculty of Mathematics and Computer,

Shahid Bahonar University of Kerman,

Kerman, Iran.

email:a.jamalizadeh@uk.ac.ir 
